\title{
Antifungal Activity of Natural and Synthetic Amides from Piper species
}

\author{
Joaquim V. Marques, ${ }^{a}$ Alberto de Oliveira, ${ }^{a, b}{ }_{\text {Ludmila Raggi, }{ }^{c} \text { Maria C. M. Young }{ }^{c} \text { and }}$ \\ Massuo J. Kato* $*$ \\ ${ }^{a}$ Instituto de Química, Universidade de São Paulo, CP 26077, 05513-970 São Paulo-SP, Brazil \\ ${ }^{b}$ Instituto de Química, Universidade Federal de Uberlândia, CP 593, 38400-902 Uberlândia-MG, Brazil \\ ${ }^{c}$ Seção de Fisiologia e Bioquímica de Plantas, Instituto de Botânica, CP 4005, 10051 São Paulo-SP, Brazil
}

\begin{abstract}
Extrato de folhas de Piper scutifolium apresentou atividade antifúngica significativa contra Cladosporium cladosporioides e C. sphaerospermum e seus principais componentes ativos, piperina, piperlonguminina e corcovadina foram isolados por meio de purificação biomonitorada, apresentando limites de detecção de $1 \mu \mathrm{g}$. Foi realizado um estudo da relação estrutura-atividade baseado na síntese de doze análogos com variações no número de insaturações, no padrão de substituição no anel aromático e no grupo amídico. As amidas sem substituintes no anel aromático e com apenas uma ligação dupla foram as mais ativas e os derivados $N, N$-dietil-substituídos apresentam maior dose-dependência.
\end{abstract}

The antifungal leaves extract from Piper scutifolium was submitted to bioactivity-guided chromatographic separation against Cladosporium cladosporioides and C. sphaerospermum yielding piperine, piperlonguminine and corcovadine as the active principles which displayed a detection limit of $1 \mu \mathrm{g}$. Structure-activity relationships were investigated with the preparation of twelve analogs having differences in the number of unsaturations, aromatic ring substituents and in the amide moiety. Analogs having a single double-bond and no substituent in the aromatic ring displayed higher activity, while $N, N$,-diethyl analogs displayed higher dose-dependent activity.

Keywords: Piper, antifungal, analogs, amides

\section{Introduction}

Piper (Piperaceae) are commonly herbs, shrubs or infrequently trees with over 1000 species described so far mostly on tropical regions. ${ }^{1}$ In addition to the high economical value of black pepper ( $P$. nigrum) as a spice, there are several medicinal uses described for different Piper species, including anti-inflammatory, analgesic and treatment of snake-bite. ${ }^{2,3}$ Several Piper species have been phytochemicaly investigated ${ }^{4-6}$ and a plethora of secondary metabolites has been found including kavalactones, ${ }^{7.8}$ lignoids, ${ }^{9-11}$ chromenes, ${ }^{12,13}$ terpenes, ${ }^{14,15}$ prenylated benzoic acids ${ }^{13,16,17}$ and also amides as the most characteristic classes of compounds. ${ }^{18,19}$

The most prominent example of a Piper amide is piperine, the pungent principle of black pepper (P. nigrum). It was the first natural product isolated from Piper species back in 1819. Piperine and several other amides have shown a variety of biological activity, e.g., antitumoral, ${ }^{20-22}$

*e-mail: majokato@iq.usp.br efflux-pump inhibitor, ${ }^{23-25}$ insecticidal ${ }^{19,26-36}$ and antifungal activity. ${ }^{37-39}$ The importance of insecticidal activity of Piper amides is considerable and thus several studies have been addressed to investigate possible structureactivity relationships. For instance, isobutylamides have shown high activity against different insects including Musca domestica, ${ }^{40}$ Aedes aegypti ${ }^{36}$ and $A$. togoi ${ }^{41}$ and compounds bearing methylenedioxyphenyl substituents can act in some cases as synergists for other compounds by interfering with cytochrome $\mathrm{P} 450$-mediated detoxifications of insecticides. ${ }^{34,42,43}$ In spite of the occurrence of several antifungal amides in plants, ${ }^{37,38,44,45}$ the data concerning the mechanism of action for such compounds is scarce and thus a comprehensive structure-activity relationship studies could not be performed so far.

In the course of bioprospecting studies for antifungal compounds from Piperaceae species, ${ }^{12,37,46-48}$ leaf extracts from $P$. scutifolium displayed significant activity against the phytopathogenic fungi Cladosporium cladosporioides and $C$. sphaerospermum. The dereplication of extracts by 
<smiles>CC(C)CNC(=O)/C=C/C=C/c1ccc2c(c1)OCO2</smiles><smiles>COC(=O)C(C)(C)CNC(=O)/C=C/C=C/c1ccc2c(c1)OCO2</smiles><smiles>O=C(/C=C/C=C/c1ccc2c(c1)OCO2)N1CCCCC1</smiles>

Figure 1. Natural amides.

a combined chromatographic and bioautographic method yielded the amides piperine (4), piperlonguminine (2e) and corcovadine (5) (Figure 1) as the major bioactive compounds. Since the detection limit of $1 \mu \mathrm{g}$ was comparable to that of positive controls miconazole and nystatin, several analogs were synthesized in order to evaluate preliminary structure-activity relationships.

\section{Experimental}

\section{General experimental procedures}

UV spectra were recorded in a UV/Visible Shimadzu UV-1601PC spectrophotometer using $\mathrm{CHCl}_{3}$ as solvent. IR spectra were obtained in a Perkin-Elmer model 1750 spectrometer. ${ }^{1} \mathrm{H}$ NMR spectra were recorded at 200 and $300 \mathrm{MHz}$ and ${ }^{13} \mathrm{C} \mathrm{NMR}$ at 50 and $75 \mathrm{MHz}$ in Bruker DPX-200 and DRX-300 spectrometers, respectively. $\mathrm{CDCl}_{3}$ (Aldrich) was used as solvent and TMS (Aldrich) as internal standard. Chemical shifts are reported in $\delta$ units (ppm) and coupling constants $(J)$ in Hz. GCLREIMS were measured in a Shimadzu GC-17A chromatograph interfaced with a MS-QP-5050A mass spectrometer. Temperature programming was performed from 150 to $300{ }^{\circ} \mathrm{C}$ at $15^{\circ} \mathrm{C} \mathrm{min}^{-1}$, then isothermal at $300^{\circ} \mathrm{C}$ for $5 \mathrm{~min}$. The injector and detector temperatures were 150 and $320^{\circ} \mathrm{C}$, respectively, and helium was used as a carrier gas. Analytical HPLC was performed using a Dionex C18 $(150 \times 5 \mathrm{~mm}$ i.d. $\times 3 \mu \mathrm{m})$ column with UVD-DAD $340 \mathrm{U}$ as a detector. Silica gel (Merck, 230-400 mesh) and Sephadex LH-20 (Amersham Biosciences) were used for column chromatographic separation, while silica gel $60 \mathrm{PF}_{254}$ (Merck) was used for analytical $(0.25 \mathrm{~mm})$ and preparative TLC $(1.0 \mathrm{~mm})$.

\section{Plant material}

P. scutifolium Jack. leaves were collected in Ubatuba, SP, Brazil, in September 2002 and identified by
Dr. Elsie Franklin Guimarães (Instituto de Pesquisas Jardim Botânico do Rio de Janeiro). Voucher specimen (Kato-281) was deposited at the Herbarium of the Jardim Botânico do Rio de Janeiro, RJ, Brazil. Dried fruits of P. nigrum were purchased in the local market.

\section{Extraction and isolation}

Dried and powdered leaves of $P$. scutifolium (10 g) were exhaustively extracted with $\mathrm{CH}_{2} \mathrm{Cl}_{2}$. Crude extract was fractionated through successive chromatography as previously described to yield piperlonguminine (4 mg, mp 157-160 ${ }^{\circ} \mathrm{C}$ ) and corcovadine $(10 \mathrm{mg}$, mp 143-145 $\left.{ }^{\circ} \mathrm{C}\right) .{ }^{47}$ To obtain piperine, dried fruits of P. nigrum $(1 \mathrm{~kg})$ were crushed, extracted with ethanol and yielded a crystaline solid after concentration under vacuum. Successive recrystallization in $\mathrm{EtOH}$ yielded pure piperine $\left(200 \mathrm{mg}\right.$, mp $\left.129-131{ }^{\circ} \mathrm{C}\right)$ which was identified based on comparison of ${ }^{1} \mathrm{H}$ and ${ }^{13} \mathrm{C}$ NMR data with those reported. ${ }^{49}$

\section{Synthetic procedures}

Compounds 1c, 1d, 2c, $2 \mathrm{~d}$ e $\mathbf{3 c}$ were synthesized using Wadsworth-Emmons according to Strunz and Finlay's method..$^{50}$ The starting aldehydes reacted with triethylphosphonocrotonate yielding ethyl 5-phenylpentadienoates. The ester products were then hydrolysed without further purification to the corresponding carboxylic acid, (2E,4E)-5-phenylpenta-2,4-dienoic acid (6) and $(2 E, 4 E)$ 5-(3-methoxyphenyl)penta-2,4-dienoic acid (7). Their corresponding chlorides obtained by reaction with oxalyl chloride reacted with amines yielding the desired amides with an overall yield of $c a .80 \%$. Compounds $\mathbf{1 d}$ and $\mathbf{2 d}$ were synthesized according to de Paula and co-workers, ${ }^{32}$ with piperic acid obtained from piperine hydrolysis which was then converted into the desired amides similarly as above described. Amides 1a, 2a and 3c were prepared 
using commercial cinnamic acid. Amides $\mathbf{1 b}$ and $\mathbf{3 b}$ were synthesized from commercial benzo[d][1,3]dioxole5-carbaldehyde (8) which was converted into (E)-3(benzo[d][1,3]dioxol-5-yl) acrylic acid (9), yielding the amides as above described. ${ }^{51}$

Spectroscopic data of 1a, 1e, 2a, 3e, $\mathbf{6}$ and $\mathbf{9}$ were in accordance with those published. ${ }^{32,51-54}$ Compounds $\mathbf{1 d}$ and $3 \mathbf{c}$ were not previously described.

(E)-3-(Benzo[d][1,3]dioxol-5-yl)-N,N-diethylacrylamide $(\mathbf{l} b)^{55}$

Yield 34\%, colorless oil; IR $v_{\max } / \mathrm{cm}^{-1} 3457,2932$, 2901, 1646, 1598, 1503, 1491, 1038, 929, 811, $523(\mathrm{KBr})$; ${ }^{1} \mathrm{H} \mathrm{NMR}\left(\mathrm{CDCl}_{3}, 200 \mathrm{MHz}\right) \delta 1.18$ (t, 3H, J 7.0 Hz, H2"a), 1.25 (t, 3H, J 7.0 Hz, H2”b), 3.40-3.50 (m, 4H, H1"a and $\mathrm{H1}$ "b), 5.98 (s, 2H, OCH $\left.\mathrm{O}_{2} \mathrm{O}\right), 6.65(\mathrm{~d}, 1 \mathrm{H}, J 15.4 \mathrm{~Hz}$, H2), 6.79 (d, 1H, J $8.0 \mathrm{~Hz}, \mathrm{H} 6$ '), 7.00 (d, 1H, J $8.0 \mathrm{~Hz}$, H5'), 7.03 (s, 1H, H2'), 7.62 (d, 1H, J 15.4 Hz, H3); ${ }^{13} \mathrm{C}$ NMR ( $\left.\mathrm{CDCl}_{3}, 50 \mathrm{MHz}\right) \delta 13.1$ (C2”a), 14.9 (C2”b), 40.9 (C1”a), 42.0 (C1'b), $101.3\left(\mathrm{OCH}_{2} \mathrm{O}\right), 106.2$ (C2'), 108.3 (C5'), 115.6 (C2), 123.5 (C6'), 129.7 (C1'), 141.8 (C3), 148.0 (C4'), 148.7 (C3'), 165.6 (C=O); EIMS m/z $247\left(\mathrm{M}^{+}, 24 \%\right), 176$ (18), 175 (75), 145 (56), 117 (35), 89 (100), 87 (11), 72 (28), 65 (14), 63 (58), 62 (22), 44 (26), 42 (36), 39 (33).

(2E,4E)-N,N-Diethyl-5-phenylpenta-2,4-dienamide $(1 c)^{56}$

Yield 57\%, colorless oil; IR $v_{\max } / \mathrm{cm}^{-1} 3349,2976,1711$, 1634, 1450, 1268, 1216, 1127 (KBr); ${ }^{1} \mathrm{H}$ NMR $\left(\mathrm{CDCl}_{3}\right.$, $300 \mathrm{MHz}) \delta 1.17$ (t, 3H, J 6.9 Hz, H2”a), 1.23 (t, 3H, $J 6.9$ Hz, H2”b), 3.42 (q, 2H, J 6.9 Hz, H1"a), 3.47 (q, 2H, $J 6.9$ Hz, H1"b), 6.41 (d, 1H, J 14.5 Hz, H2), 6.80-7.00 (m, 2H, H4 and H4'), 7.24-7.56 (m, 6H, H2', H8, H5', H6', H5 and $\mathrm{H} 3) ;{ }^{13} \mathrm{C} \mathrm{NMR}\left(\mathrm{CDCl}_{3}, 75 \mathrm{MHz}\right) \delta 13.2(\mathrm{C} 2 " \mathrm{a}), 15.0$ (C2”b), 41.0 (C1"a), 42.2 (C1"b), 121.3 (C2), 126.3 (C4), 127.0 (C2' and C6'), 128.6 (C4'), 128.7 (C3' and C5'), 136.5 (C1'), 138.7 (C5), 142.4 (C3), 165.8 (C1); EIMS $\mathrm{m} / \mathrm{z} 157$ (12\%), 129 (16), 128 (43), 127 (22), 102 (17), 77 (45), 72 (32), 56 (24), 51 (36), 42 (100), 39 (23).

(2E,4E)-N,N-Diethyl-5-(3-methoxyphenyl)penta-2,4dienamide (1d)

Yield 72\%, colorless oil; IR $v_{\max } / \mathrm{cm}^{-1} 3405,2974,2937$, 2916, 1632, 1605, 1484, 1462, 1264, 1044, 784, 687 (KBr); ${ }^{1} \mathrm{H} \mathrm{NMR}\left(\mathrm{CDCl}_{3}, 200 \mathrm{MHz}\right) \delta 1.13$ (t, 3H, J 7.4 Hz, H2"a), 1.23 (t, 3H, J 7.4 Hz, H2"b), 3.40 (t, 2H, J 7.4 Hz, H1"a), 3.52 (t, 2H, J $7.4 \mathrm{~Hz}, \mathrm{H1}$ 'b), 3.74 (s, 3H, $\mathrm{OCH}_{3}$ ), 6.41 (d, $1 \mathrm{H}, J 15.0 \mathrm{~Hz}, \mathrm{H} 2)$, 6.69-6.84 (m, 3H , H4', H5 and H4), 6.89 (s, 1H, H2'), 6.97 (d, 1H, J 7.9 Hz, H6'), 7.18 (t, 1H, $J 7.9 \mathrm{~Hz}, \mathrm{H} 5$ ') 7.38 (dd, $1 \mathrm{H}, J 15.0 \mathrm{~Hz}$ and $9.1 \mathrm{~Hz}, \mathrm{H} 3$ ); ${ }^{13} \mathrm{C}$ NMR ( $\left.\mathrm{CDCl}_{3}, 50 \mathrm{MHz}\right) \delta 13.2$ (C2"a), 14.9 (C2”b),
41.0 (C1"a), 42.2 (C1'b), $55.2\left(\mathrm{OCH}_{3}\right), 111.9$ (C2'), 114.4 (C4'), 119.6 (C6'), 121.0 (C2), 127.2 (C5), 129.7 (C5'), 137.8 (C1'), 138.7 (C4), 142.4 (C3), 159.8 (C3'), 165.8 (C1); EIMS m/z 135 (5\%), 100 (10), 72 (55), 57 (30), 55 (18), 44 (66), 43 (100), 42 (64), 41 (72).

(2E,4E)-N-Isobutyl-5-phenylpenta-2,4-dienamide $(2 c)^{57}$

Yield 79\%, white crystals, mp 154.2-154.9 ${ }^{\circ} \mathrm{C}$; IR $v_{\text {max }} / \mathrm{cm}^{-1} 3292,2961,1646,1612,1546,1446,1346$, $1160,989,508(\mathrm{KBr}) ;{ }^{1} \mathrm{H} \mathrm{NMR}\left(\mathrm{CDCl}_{3}, 300 \mathrm{MHz}\right) \delta 0.94$ (d, 6H, J 6.8 Hz, H3"), 1.82 (hept, 1H, J 6.8 Hz, H2"), 3.19 (t, 2H, J 6.6 Hz, H1"), 5.71 (sl, 1H, NH), 5.98 (d, 1H, $J 14.9 \mathrm{~Hz}, \mathrm{H} 2$ ), 6.83-6.88 (m, 2H, H4 and H4'), 7.24-7.50 (m, 6H, H2', H3', H5', H6', $\mathrm{H} 5$ and $\mathrm{H} 3)$; ${ }^{13} \mathrm{C} \mathrm{NMR}\left(\mathrm{CDCl}_{3}\right.$, $75 \mathrm{MHz}$, ) $\delta 20.1$ (C3”), 28.6 (C2”), 47.0 (C1”), 124.1 (C2), 126.3 (C4), 126.9 (C2' and C6'), 128.6 (C4'), 128.7 (C3' and C5'), 136.3 (C4'), 139.0 (C5), 140.8 (C3), 166.1 (C1); EIMS $m / z 229\left(\mathrm{M}^{+}, 34 \%\right), 172$ (13), 158 (12), 157 (100), 129 (35), 128 (98), 96 (42), 41 (24).

(2E,4E)-N-Isobutyl-5-(3-methoxyphenyl)penta-2,4dienamide $(\mathbf{2 d})^{58}$

Yield $82 \%$, white crystals, mp 99.5-101.0 ${ }^{\circ} \mathrm{C}$, IR $v_{\text {max }} / \mathrm{cm}^{-1} 3308,2950,1644,1609,1578,1432,1161$, 1052, 990, 863, $572(\mathrm{KBr}) ;{ }^{1} \mathrm{H} \mathrm{NMR}\left(\mathrm{CDCl}_{3}, 300 \mathrm{MHz}\right)$ $\delta 0.94$ (d, 6H, J 6.6, H3"), 1.83 (m, 1H, J 6.6 Hz, H2"), 3.19 (t, 2H, J 6.6 Hz, H1"), 3.81 (s, 3H, $\mathrm{OCH}_{3}$ ), 5.99 (d, $1 \mathrm{H}, J 14.5 \mathrm{~Hz}, \mathrm{H} 2$ ), 6.75-6.90 (m, 3H, H4', H5 and H4), 6.95 (t, 1H, J 2.0 Hz, H2'), 7.03 (d, 1H, J 7.9 Hz, H6'), 7.24 (t, 1H, J 7.9 Hz, H5'), 7.53 (m, 1H, H3); ${ }^{13} \mathrm{C}$ NMR $\left(\mathrm{CDCl}_{3}, 75 \mathrm{MHz}\right) \delta 20.1$ (C3”), 28.6 (C2"), 47.0 (C1"), $55.2\left(\mathrm{OCH}_{3}\right), 112.1$ (C4'), 114.3 (C2'), 119.6 (C6'), 124.2 (C2), 126.6 (C4), 129.7 (C5'), 137.7 (C1'), 138.9 (C5), 140.7 (C3), 159.8 (C3'), 166.0 (C1); EIMS m/z 259 ( $\mathbf{M}^{+}$, 4\%), 187 (35), 160 (11), 158 (71), 145 (12), 144 (52), 115 (88), 77 (11), 64 (10), 43 (100), 41 (90), 39 (41).

\section{$N$-Pentylcinnamamide $(\mathbf{3 a})^{59}$}

Yield 95\%, white crystals, mp 87.6-88.2 ${ }^{\circ} \mathrm{C}$; IR $v_{\text {max }} / \mathrm{cm}^{-1} 3276,2955,2933,1653,1607,1553,1475$, 764, 489 (KBr); ${ }^{1} \mathrm{H}$ NMR $\left(\mathrm{CDCl}_{3}, 200 \mathrm{MHz}\right) \delta 0.86$ (brm, 3H, H5"), 1.29 (brm, 4H, H3" and H4”), 1.59 (brm, 2H, H2"), 3.38 (q, 2H, J 6.6 Hz, H1"), 6.68 (d, 1H, J $15.8 \mathrm{~Hz}$, $\mathrm{H} 2), 7.20-7.35$ (m, 4H, H3', H4', H5' and NH), 7.41-7.46 (m, 2H, H2' and H6'), $7.64(\mathrm{~d}, 1 \mathrm{H}, J 15.8 \mathrm{~Hz}, \mathrm{H} 3)$;

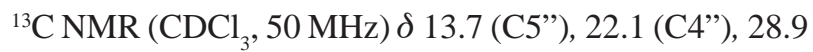
(C3"), 29.0 (C2"), 39.6 (C1"), 121.3 (C2), 127.4 (C2' and C4'), 128.4 (C3' and C5'), 129.1 (C4'), 134.7 (C1'), 139.9 (C3), $166.3(\mathrm{C}=\mathrm{O})$; EIMS m/z $217\left(\mathrm{M}^{+}, 4 \%\right), 160$ (13), 146 (25), 131 (100), 103 (49), 102 (16), 84 (18), 77 (58), 51 (24), 41 (28), 39 (15). 
(E)-3-(Benzo[d][1,3]dioxol-5-yl)-N-pentylacrylamide $(3 \boldsymbol{b})^{55}$

Yield $47 \%$, orange crystals, mp $99.8-100.2{ }^{\circ} \mathrm{C}$; IR $v_{\max } / \mathrm{cm}^{-1} 3298,2934,1655,1621,1504,1491,97,927$ (KBr); ${ }^{1} \mathrm{H} \mathrm{NMR}\left(\mathrm{CDCl}_{3}, 200 \mathrm{MHz}\right) \delta 0.79$ (brm, 3H, H5"), 1.24 (brm, 4H, H3" and H4"), 1.48 (brm, 2H, H2"), 3.28 (q, 2H, J 6.7 Hz, H1"), $5.86\left(\mathrm{~s}, 2 \mathrm{H}, \mathrm{OCH}_{2} \mathrm{O}\right), 6.25$ (d, 1H, $J 15.4 \mathrm{~Hz}, \mathrm{H} 2), 6.36(\mathrm{sl}, 1 \mathrm{H}, \mathrm{NH}), 6.66(\mathrm{~d}, 1 \mathrm{H}, J 8.0 \mathrm{~Hz}$, H6'), 6.85 (d, 1H, J 8.0 Hz, H5'), 6.88 (s, 1H, H2'), 7.43 $(\mathrm{d}, 1 \mathrm{H}, J 15.4 \mathrm{~Hz}, \mathrm{H} 3) ;{ }^{13} \mathrm{C} \mathrm{NMR}\left(\mathrm{CDCl}_{3}, 50 \mathrm{MHz}\right) \delta 13.8$ (C5"), 22.3 (C4”), 29.0 (C3"), 29.2 (C2"), 39.7 (C1"), $101.2\left(\mathrm{OCH}_{2} \mathrm{O}\right), 106.2\left(\mathrm{C}^{\prime}\right), 108.3\left(\mathrm{C}^{\prime}\right), 119.1(\mathrm{C} 2)$, 123.6 (C6'), 129.2 (C1'), 140.1 (C3), 148.0 (C4'), 148.8 (C3'), $166.2(\mathrm{C}=\mathrm{O})$; EIMS m/z $261\left(\mathrm{M}^{+}, 19 \%\right), 190$ (53), 176 (35), 175 (89), 145 (56), 135 (40), 117 (29), 89 (100), 41 (58), 39 (43).

\section{(2E,4E)-N-Pentyl-5-phenylpenta-2,4-dienamide (3c)}

Yield $80 \%$, yellowish crystals, mp 103.9-104.3 ${ }^{\circ} \mathrm{C}$; $\mathrm{IR} v_{\text {max }} / \mathrm{cm}^{-1} 3287,2958,1644,1615,1547,1443,1146$, $998(\mathrm{KBr}) ;{ }^{1} \mathrm{H}$ NMR $\left(200 \mathrm{MHz}, \mathrm{CDCl}_{3}\right) \delta 0.90(\mathrm{t}, 3 \mathrm{H}$, $J 6.6 \mathrm{~Hz}, \mathrm{H} 5$ "), 1.25-1.36 (m, 4H, H3" and H4"), 1.55 (t, 2H, J 6.6 Hz, H2"), 3.29 (q, 2H, J 6.6 Hz, H1"), 5.77 (sl, $1 \mathrm{H}, \mathrm{NH}), 5.98$ (d, 1H, J $14.9 \mathrm{~Hz}, \mathrm{H} 2), 6.80-6.89$ (m, 2H, H4 and H5), 7.24-7.50 (m, 6H, H3, H2', H3', H4', H5' and $\mathrm{H} 6$ '); ${ }^{13} \mathrm{C} \mathrm{NMR}\left(\mathrm{CDCl}_{3}, 50 \mathrm{MHz}\right) \delta 13.9$ (C5”), 22.3 (C4"), 29.0 (C3”), 29.2 (C2”), 39.6 (C1”), 124.3 (C4), 126.3 (C2), 126.9 (C2' and C6'), 128.5 (C4'), 128.6 (C3' and C3'), 136.2 (C1'), 138.8 (C5), 140.4 (C3), 166.1 (C1); EIMS $\mathrm{m} / \mathrm{z} 243\left(\mathrm{M}^{+}, 28 \%\right), 186(17), 157$ (90), 130 (23), 129 (58), 128 (100), 127 (45), 115 (16), 96 (41), 91 (11), 77 (22), 64 (22), 51 (17), 43 (20), 41 (35), 39 (18).

(2E,4E)-5-(3-Methoxyphenyl)penta-2,4-dienoic acid (7) ${ }^{60}$

Yield 88\%, colorless needles, mp 135.9-136.6 ${ }^{\circ} \mathrm{C}$; IR $v_{\max } / \mathrm{cm}^{-1} 3025,3007,1687,1612,1579,1426,1158$, 1044, 995, 873 (KBr); ${ }^{1} \mathrm{H}$ NMR $\left(\mathrm{CDCl}_{3}, 300 \mathrm{MHz}\right) \delta 3.84$ (s, 3H, $\left.\mathrm{OCH}_{3}\right), 5.99$ (d, 1H, J 15.2 Hz, H2), 6.85-6.95 (m, 2H, H4' and H4), 6.93 (d, 1H, J 15.7 Hz, H5), 6.99 (t, 1H, $\left.J 2.5 \mathrm{~Hz}, \mathrm{H} 2^{\prime}\right), 7.07$ (d, 1H, J 7. $8 \mathrm{~Hz}, \mathrm{H6}$ '), 7.28 (t, 1H, $J 7.8 \mathrm{~Hz}, \mathrm{H} 5$ '), 7.53 (ddd, $J$ 15.2, 7.5 and $2.3 \mathrm{~Hz}, 1 \mathrm{H}, \mathrm{H} 3$ ); ${ }^{13} \mathrm{C} \mathrm{NMR}\left(\mathrm{CDCl}_{3}, 75 \mathrm{MHz}\right) \delta 55.3\left(\mathrm{OCH}_{3}\right), 112.3\left(\mathrm{C}^{\prime}\right)$, 115.1 (C2'), 120.1 (C6'), 120.4 (C2), 126.2 (C4), 129.8 (C5'), 137.2 (C1'), 141.54 (C5), 146.9 (C3), 159.9 (C3'), 172.3 (C1); EIMS m/z $204\left(\mathrm{M}^{+}, 65 \%\right), 159$ (98), 144 (100), 127 (40), 115 (75), 89 (12), 77 (14), 63 (20), 51 (17), 39 (19).

\section{Antifungal assay}

The microorganisms used in the antifungal assay, Cladosporium cladosporioides (Fresen) de Vries SPC 140 and C. sphaerospermum (Perzig) SPC 491, have been maintained at the Instituto de Botânica, São Paulo, SP, Brazil. The assay was carried out for all amides and their activities determined as previously described (Table 3). ${ }^{47}$ Nystatin and miconazole were used as positive controls whereas ampicillin and chloramphenicol were used as negative controls. ${ }^{61}$

\section{Results and Discussion}

Natural amides were isolated through successive chromatographic procedures as previously described. ${ }^{47}$ Analogs of $(2 E, 4 E)$-5-phenylpenta-2,4-dienamides and $(E)$-cinnamamides were synthesized (Figure 2) aiming at determination of overall effect of aromatic ring substitution and nitrogen substituent in antifungal activity. ${ }^{62,63}$ The importance of the amide moiety for the antifungal activity was investigated by replacing the natural piperidyl, isobutyl or its acetylated derivative by $\mathrm{N}, \mathrm{N}$-diethyl or $\mathrm{N}$-pentyl analogs.

The replacement of isobutyl or piperidyl moieties by diethyl groups in (2E, 4E)-5-phenylpenta-2,4-dienamides resulted in a noticeable increase in the dose-response activity, as observed for amides $\mathbf{1 a}, \mathbf{1 b}, \mathbf{1 c}, \mathbf{1 d}$ and $\mathbf{1 e}$ against $C$. cladosporioides and C. sphaerospermum. Some selectivity was detected between the two strains in which $C$. sphaerospermum was more sensible to $1 \mathrm{e}$ than C. cladosporioides (Table 1).

Analysis of aromatic substitution pattern indicated that amides having methylenedioxy or methoxyl groups displayed lower antifungal potency when compared to those having no substituents. The amides $\mathbf{2 a}, \mathbf{2 c}, \mathbf{3 a}$ and $3 \mathbf{c}$ displayed higher activities at $1 \mu \mathrm{g}$ against both strains while $\mathbf{1 d}, \mathbf{3 b}$ and $\mathbf{3 e}$ were the least active among all amides assayed against $C$. cladosporioides. At lower concentrations $N$-isobutyl and $N$-pentyl derivatives (2a-2e, $\mathbf{3 a - 3}$ c and $\mathbf{3 e}$ ) showed higher activity. In this case, there is an apparent positive correlation with the lipophylicity and the amides having a $\alpha, \beta$-conjugated carbonyl were more active than those having an extended $\alpha, \beta, \gamma, \delta$-conjugated system.

\section{Conclusions}

Investigation of natural and synthetic amides as antifungal compounds have shown preliminary structureactivity relationship in which $N, N$-diethyl showed higher dose dependent activity while $N$-pentyl and $N$-isobutyl derivatives showed lower detection limit. In general, substituents in the aromatic ring such as methoxyl and methylenedioxy decreased antifungal activity and the shorter aliphatic chain, the higher activity was observed. 
Table 1. Antifungal activity of natural and synthetic compounds

\begin{tabular}{|c|c|c|c|c|c|c|c|c|c|c|c|c|c|c|c|c|c|}
\hline & \multirow[t]{2}{*}{$\mathrm{n}$} & \multirow[t]{2}{*}{$\mathrm{R}^{1}$} & \multirow[t]{2}{*}{$\mathrm{R}^{2}$} & \multirow[t]{2}{*}{$\mathrm{R}^{3}$} & \multirow[t]{2}{*}{$\mathrm{R}^{4}$} & \multicolumn{6}{|c|}{ Cladosporium cladosporioides ${ }^{\#}$} & \multicolumn{6}{|c|}{ Cladosporium sphaerospermum ${ }^{\#}$} \\
\hline & & & & & & 100 & 50 & 25 & 10 & 5 & 1 & 100 & 50 & 25 & 10 & 5 & 1 \\
\hline $1 \mathbf{a}$ & 1 & $\mathrm{H}$ & $\mathrm{H}$ & Et & $\mathrm{Et}$ & $* * *$ & $* * *$ & $* * *$ & $* *$ & $* *$ & $*$ & $* * *$ & $* * *$ & $* * *$ & $* *$ & $* *$ & - \\
\hline $1 \mathrm{~b}$ & 1 & \multicolumn{2}{|c|}{$\mathrm{O}_{2} \mathrm{CH}_{2}$} & Et & Et & $* * *$ & $* * *$ & $* * *$ & $* *$ & $* *$ & $*$ & $* * *$ & $* * *$ & $* * *$ & $* * *$ & $* *$ & $*$ \\
\hline $1 \mathrm{c}$ & 2 & $\mathrm{H}$ & $\mathrm{H}$ & Et & Et & $* * *$ & $* * *$ & $* * *$ & $* * *$ & $* *$ & $*$ & $* * *$ & $* * *$ & $* * *$ & $* *$ & $*$ & - \\
\hline 1d & 2 & $\mathrm{OMe}$ & $\mathrm{H}$ & Et & Et & $* * *$ & $* * *$ & $* *$ & $*$ & $*$ & - & $* * *$ & $* * *$ & $* *$ & $*$ & $*$ & - \\
\hline 1e & 2 & \multicolumn{2}{|c|}{$\mathrm{O}_{2} \mathrm{CH}_{2}$} & Et & Et & $* *$ & $* *$ & $* *$ & $*$ & $*$ & - & $* * *$ & $* * *$ & $* *$ & $*$ & $*$ & $*$ \\
\hline $2 a$ & 1 & $\mathrm{H}$ & $\mathrm{H}$ & ${ }^{i} \mathrm{Bu}$ & $\mathrm{H}$ & $* *$ & $* *$ & $* *$ & $* *$ & $* *$ & $* *$ & $* *$ & $* *$ & $* *$ & $* *$ & $* *$ & $*$ \\
\hline $2 c$ & 2 & $\mathrm{H}$ & $\mathrm{H}$ & ${ }^{i} \mathrm{Bu}$ & $\mathrm{H}$ & $* *$ & $* *$ & $* *$ & $* *$ & $* *$ & $*$ & $* *$ & $* *$ & $* *$ & $* *$ & $* *$ & $* *$ \\
\hline $2 d$ & 2 & $\mathrm{OMe}$ & $\mathrm{H}$ & ${ }^{i} \mathrm{Bu}$ & $\mathrm{H}$ & $* *$ & $* *$ & $* *$ & $* *$ & $*$ & $*$ & $* *$ & $* *$ & $* *$ & $* *$ & $*$ & $*$ \\
\hline$\underline{2 e}$ & 2 & \multicolumn{2}{|c|}{$\mathrm{O}_{2} \mathrm{CH}_{2}$} & ${ }^{i} \mathrm{Bu}$ & $\mathrm{H}$ & $* *$ & $* *$ & $* *$ & $* *$ & $* *$ & $*$ & $* *$ & $* *$ & $* *$ & $* *$ & $* *$ & $*$ \\
\hline $3 \mathbf{a}$ & 1 & $\mathrm{H}$ & $\mathrm{H}$ & pentyl & $\mathrm{H}$ & $* *$ & $* *$ & $* *$ & $* *$ & $* *$ & $* *$ & $* *$ & $* *$ & $* *$ & $* *$ & $* *$ & $* *$ \\
\hline $3 \mathbf{b}$ & 1 & \multicolumn{2}{|c|}{$\mathrm{O}_{2} \mathrm{CH}_{2}$} & pentyl & $\mathrm{H}$ & $*$ & $*$ & $*$ & $*$ & $*$ & $*$ & $* *$ & $* *$ & $* *$ & $*$ & $*$ & $*$ \\
\hline $3 \mathbf{c}$ & 2 & $\mathrm{H}$ & $\mathrm{H}$ & pentyl & $\mathrm{H}$ & $* *$ & $* *$ & $* *$ & $* *$ & $* *$ & $* *$ & $* *$ & $* *$ & $* *$ & $* *$ & $* *$ & $* *$ \\
\hline $3 \mathbf{e}$ & 2 & \multicolumn{2}{|c|}{$\mathrm{O}_{2} \mathrm{CH}_{2}$} & pentyl & $\mathrm{H}$ & $*$ & $*$ & $*$ & $*$ & $*$ & - & $*$ & $*$ & $*$ & $*$ & $*$ & $*$ \\
\hline 4 & 2 & \multicolumn{2}{|c|}{$\mathrm{O}_{2} \mathrm{CH}_{2}$} & piperidyl & $* *$ & $* *$ & $* *$ & $* *$ & $*$ & $*$ & $* *$ & $* *$ & $* *$ & $* *$ & $* *$ & $* *$ & \\
\hline$\underline{5}$ & 2 & \multicolumn{2}{|c|}{$\mathrm{O}_{2} \mathrm{CH}_{2}$} & $\mathrm{CH}_{2} \mathrm{C}\left(\mathrm{OCOCH}_{3}\right)\left(\mathrm{CH}_{3}\right)_{2}$ & $\mathrm{H}$ & $* *$ & $* *$ & $* *$ & $* *$ & $* *$ & $*$ & $* *$ & $* *$ & $* *$ & $* *$ & $* *$ & $*$ \\
\hline
\end{tabular}

Numbers in italic and underlined are natural amides; ${ }^{*} \mu \mathrm{g}$; ${ }^{* * *}$ : high activity; ${ }^{* *}$ : medium; * : low; - : not active.<smiles>[R]CNC(=O)/C=C/c1ccc([R2])c([R])c1</smiles>

la: $n=1 ; R^{1}=R^{2}=H ; R^{3}=R^{4}=E t$

1b: $n=1 ; R^{1}+R^{2}=\mathrm{O}_{2} \mathrm{CH}_{2} ; R^{3}=\mathrm{R}^{4}=\mathrm{Et}$

lc: $n=2 ; R^{1}=R^{2}=H ; R^{3}=R^{4}=E t$

Id: $n=2 ; R^{1}=O M e ; R^{2}=H ; R^{3}=R^{4}=E t$

le: $n=2 ; R^{1}+R^{2}=\mathrm{O}_{2} \mathrm{CH}_{2} ; R^{3}=R^{4}=E t$

2a: $n=1 ; R^{1}=R^{2}=H ; R^{3}=i-B u ; R^{4}=H$

2c: $n=2 ; R^{1}=R^{2}=H ; R^{3}=i-B u ; R^{4}=H$

2d: $n=2 ; R^{1}=O M e ; R^{2}=H ; R^{3}=i-B u ; R^{4}=H$

2e: $n=2 ; R^{1}+R^{2}=\mathrm{O}_{2} \mathrm{CH}_{2} ; R^{3}=i-B u ; R^{4}=H$ 3a: $n=1 ; R^{1}=R^{2}=H ; R^{3}=$ pentyl; $R^{4}=H$

3b: $n=1 ; R^{1}+R^{2}=\mathrm{O}_{2} \mathrm{CH}_{2} ; R^{3}=$ pentyl; $\mathrm{R}^{4}=\mathrm{H}$

3c: $n=2 ; R^{1}=R^{2}=H ; R^{3}=$ pentyl; $R^{4}=H$

3e: $n=2 ; R^{1}+R^{2}=\mathrm{O}_{2} \mathrm{CH}_{2} ; R^{3}=$ pentyl; $\mathrm{R}^{4}=\mathrm{H}$

4: $\mathrm{n}=2 ; \mathrm{R}^{1}+\mathrm{R}^{2}=\mathrm{OCH}_{2} \mathrm{O} ; \mathrm{R}^{3}+\mathrm{R}^{4}=$ piperidyl

5: $n=2 ; R^{1}+R^{2}=\mathrm{O}_{2} \mathrm{CH}_{2} ; \mathrm{R}^{3}=\mathrm{CH}_{2} \mathrm{C}\left(\mathrm{OCOCH}_{3}\right)\left(\mathrm{CH}_{3}\right)_{2} ; \mathrm{R}^{4}=\mathrm{H}$

Figure 2. Synthetic and natural amides evaluated as antifungal.

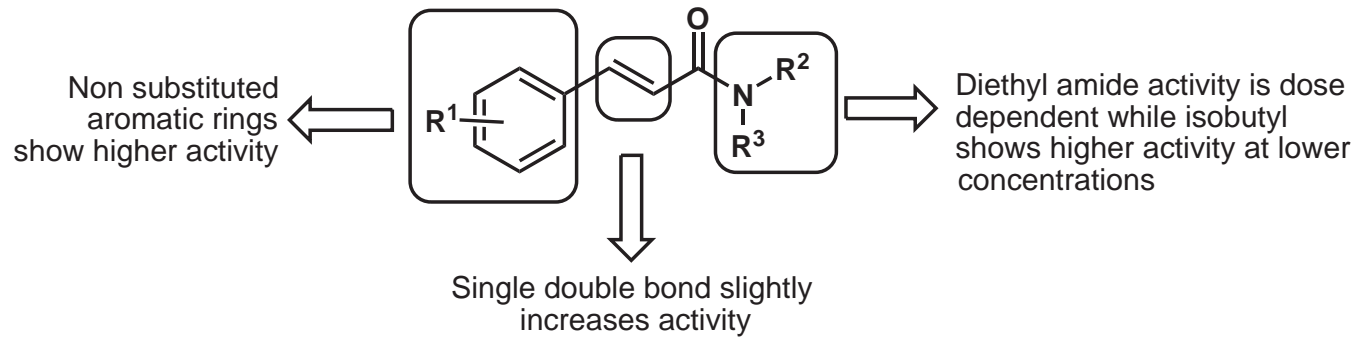

Figure 3. Preliminary structure-activity relationship for antifungal amide. 
Further investigations including quantitative biological activity assessment and determination of physicochemical descriptors are required for a thorough understanding of the observed antifungal activities and in the development of effective antifungal agents.

\section{Supplementary Information}

The spectral data are available free of charge at http://jbcs.sbq.org.br, as pdf file.

\section{Acknowledgments}

Authors thank FAPESP, CNPq and CAPES for financial support.

\section{References}

1. Jaramillo, M. A.; Manos, P. S.; Am. J. Bot. 2001, 88, 706.

2. Di Stasi, L. C.; Oliveira, G. P.; Carvalhaes, M. A.; Queiroz Jr, M.; Tien, O. S.; Kakinami, S. H.; Reis, M. S.; Fitoterapia 2002, 73,69 .

3. Giorgetti, M.; Negri, G.; Rodrigues, E.; J. Ethnopharmacol. 2007, 109, 338.

4. Kato, M. J.; Furlan, M.; Pure Appl. Chem. 2007, 79, 529.

5. Parmar, V. S.; Jain, S. C.; Gupta, S.; Talwar, S.; Rajwanshi, V. K.; Kumar, R.; Azim, A.; Malhotra, S.; Kumar, N.; Jain, R.; Sharma, N. K.; Tyagi, O. D.; Lawrie, S. J.; Errington, W.; Howarth, O. W.; Olsen, C. E.; Singh, S. K.; Wengel, J.; Phytochemistry 1998, 49, 1069.

6. Parmar, V. S.; Jain, S. C.; Bisht, K. S.; Jain, R.; Taneja, P.; Jha, A.; Tyagi, O. D.; Prasad, A. K.; Wengel, J.; Olsen, C. E.; Boll, P. M.; Phytochemistry 1997, 46, 597.

7. Xuan, T. D.; Fukuta, M.; Wei, A. C.; Elzaawely, A. A.; Khanh, T. D.; Tawata, S.; J. Nat. Med. 2008, 62, 188.

8. Whitton, P. A.; Lau, A.; Salisbury, A.; Whitehouse, J.; Evans, C. S.; Phytochemistry 2003, 64, 673.

9. Chen, Y.-C.; Liao, C.-H.; Chen, I.-S.; Phytochemistry 2007, 68 , 2101.

10. Bodiwala, H. S.; Singh, G.; Singh, R.; Dey, C. S.; Sharma, S. S.; Bhutani, K. K.; Singh, I. P.; J. Nat. Med. 2007, 61, 418.

11. Martins, R. C.; Latorre, L. R.; Sartorelli, P.; Kato, M. J.; Phytochemistry 2000, 55, 843.

12. Morandim, A. A.; Bergamo, D. C. B.; Kato, M. J.; Cavalheiro, A. J.; Bolzani, V. S.; Furlan, M.; Phytochem. Anal. 2005, 16, 282.

13. Baldoqui, D. C.; Kato, M. J.; Cavalheiro, A. J.; Bolzani, V. S.; Young, M. C. M.; Furlan, M.; Phytochemistry 1999, 51, 899.

14. Baldoqui, D. C.; Bolzani, V. S.; Furlan, M.; Kato, M. J.; Marques, M. O. M.; Quim. Nova 2009, 32, 1107.
15. Péres, V. F.; Moura, D. J.; Sperotto, A. R. M.; Damasceno, F. C.; Caramão, E. B.; Zini, C. A.; Saffi, J.; Food Chem. Toxicol. 2009, 47, 2389.

16. Lago, J. H. G.; Chen, A.; Young, M. C. M.; Guimarães, E. F.; Oliveira, A.; Kato, M. J.; Phytochem. Lett. 2009, 2, 96.

17. Ramos, C. S.; Kato, M. J.; J. Braz. Chem. Soc. 2009, 20, 560.

18. Cotinguiba, F.; Regasini, L. O.; Bolzani, V. S.; Debonsi, H. M.; Passerini, G. D.; Cicarelli, R. M. B.; Kato, M. J.; Furlan, M.; Med. Chem. Res. 2009, 18, 703.

19. Srinivasan, K.; Crit. Rev. Food Sci. Nutr. 2007, 47, 735.

20. Bezerra, D. P.; Moura, D. J.; Rosa, R. M.; Vasconcellos, M. C.; Silva, A. C. R.; Moraes, M. O.; Silveira, E. R.; Lima, M. A. S.; Henriques, J. A. P.; Costa-Lotufo, L. V.; Saffi, J.; Mutat. Res./ Genet. Toxicol. Environ. Mutagen. 2008, 652, 164.

21. Bezerra, D. P.; Pessoa, C.; Moraes, M. O.; Alencar, N. M. N.; Mesquita, R. O.; Lima, M. W.; Alves, A. P. N.; Pessoa, O. D. L.; Chaves, J. H.; Silveira, E. R.; Costa-Lotufo, L. V.; J. Appl. Toxicol. 2008, 28, 599.

22. Liu, D.; Meng, Y.; Zhao, J.; Chen, L.; Chem. Res. Chin. Univ. 2008, 24, 42 .

23. Michalet, S.; Cartier, G.; David, B.; Mariotte, A. M.; Dijoux Franca, M. G.; Kaatz, G. W.; Stavri, M.; Gibbons, S.; Bioorg. Med. Chem. Lett. 2007, 17, 1755.

24. Sangwan, P. L.; Koul, J. L.; Koul, S.; Reddy, M. V.; Thota, N.; Khan, I. A.; Kumar, A.; Kalia, N. P.; Qazi, G. N.; Bioorg. Med. Chem. 2008, 16, 9847.

25. Thota, N.; Koul, S.; Reddy, M. V.; Sangwan, P. L.; Khan, I. A.; Kumar, A.; Raja, A. F.; Andotra, S. S.; Qazi, G. N.; Bioorg. Med. Chem. 2008, 16, 6535.

26. Bezerra, D. P.; Castro, F. O.; Alves, A. P. N.; Pessoa, C.; Moraes, M. O.; Silveira, E. R.; Lima, M. A. S.; Elmiro, F. J. M.; CostaLotufo, L. V.; Braz. J. Med. Biol. Res. 2006, 39, 801.

27. Bina, S. S.; Tahsin, G.; Sabira, B.; Farhana, A.; Fouzia, A. S.; Nat. Prod. Res. 2005, 19, 143.

28. Christodoulopoulou, L.; Tsoukatou, M.; Tziveleka, L. A.; Vagias, C.; Petrakis, P. V.; Roussis, V.; J. Agric. Food Chem. 2005, 53, 1435.

29. Khan, I. A.; Mirza, Z. M.; Kumar, A.; Verma, V.; Qazi, G. N.; Antimicrob. Agents Chemother. 2006, 50, 810.

30. Lee, S. A.; Hong, S. S.; Han, X. H.; Hwang, J. S.; Oh, G. J.; Lee, K. S.; Lee, M. K.; Hwang, B. Y.; Ro, J. S.; Chem. Pharm. Bull. 2005, 53, 832.

31. Navickiene, H. M. D.; Bolzani, V. S.; Kato, M. J.; Pereira, A. M. S.; Bertoni, B. W.; França, S. C.; Furlan, M.; Phytochem. Anal. 2003, 14, 281.

32. Paula, V. F.; Barbosa, L. C. A.; Demuner, A. J.; Piló-Veloso, D.; Picanço, M. C.; Pest Manage. Sci. 2000, 56, 168.

33. Ribeiro, T. S.; Lima, L. F.; Previato, J. O.; Previato, L. M.; Heise, N.; Lima, M. E. F.; Bioorg. Med. Chem. Lett. 2004, 14, 3555.

34. Scott, I. M.; Jensen, H. R.; Philogène, B. J. R.; Arnason, J. T. A.; Phytochem. Rev. 2008, 7, 65. 
35. Brown, D.; Zhang, L.; Wen, Z.; Scott, J. G.; Arch. Insect Biochem. Physiol. 2003, 54, 212.

36. Simas, N. K.; Lima, E. C.; Kuster, R. M.; Lage, C. L. S.; Oliveira Filho, A. M.; Rev. Soc. Bras. Med. Trop. 2007, 40, 405.

37. Alecio, A. C.; Bolzani, V. S.; Young, M. C. M.; Kato, M. J.; Furlan, M.; J. Nat. Prod. 1998, 61, 637.

38. Cunico, M. M.; Miguel, O. G.; Miguel, M. D.; Carvalho, J. L. S.; Peitz, C.; Auer, C. G.; Grigoletti Jr., A.; Visão Acadêmica 2003, $4,77$.

39. Silva, R. V.; Navickiene, H. M. D.; Kato, M. J.; Bolzani, V. S.; Meda, C. I.; Young, M. C. M.; Furlan, M.; Phytochemistry 2002, 59, 479.

40. Elliott, M.; Farnham, A. W.; Janes, N. F.; Johnson, D. M.; Pulman, D. A.; Sawicki, R. M.; Agric. Biol. Chem. 1986, 50, 1347.

41. Park, I. K.; Lee, S. G.; Shin, S. C.; Park, J. D.; Ahn, Y. J.; J. Agric. Food Chem. 2002, 50, 1866.

42. Pring, B. G.; J. Chem. Soc., Perkin Trans.1 1982, 1493.

43. Scott, I. M.; Puniani, E.; Durst, T.; Phelps, D.; Merali, S.; Assabgui, R. A.; Sanchez-Vindas, P.; Poveda, L.; Philogène, B. J. R.; Arnason, J. T.; Agric. Forest Entomol. 2002, 4, 137.

44. Navickiene, H. M. D.; Alécio, A. C.; Kato, M. J.; Bolzani, V. S.; Young, M. C. M.; Cavalheiro, A. J.; Furlan, M.; Phytochemistry 2000, 55, 621 .

45. Silva, R. V.; Navickiene, H. M. D.; Kato, M. J.; Bolzani, V. S.; Meda, C. I.; Young, M. C. M.; Furlan, M.; Phytochemistry 2002, 59, 521.

46. Lago, J. G. L.; Ramos, C. S.; Casanova, C. C. D.; Morandim, A. A.; Bergamo, C. D.; Cavalheiro, A. J.; Bolzani, V. S.; Furlan, M.; Guimarães, E. F.; Young, M. C. M.; Kato, M. J.; J. Nat. Prod. 2004, 67, 1783.

47. Marques, J. V.; Kitamura, R. O. S.; Lago, J. H. G.; Young, M. C. M.; Guimarães, E. F.; Kato, M. J.; J. Nat. Prod. 2007, 70, 2036.
48. Sartorelli, P.; Young, M. C. M.; Kato, M. J.; Phytochemistry 1998, 47, 1003.

49. Chen, Z.; Wu, J. B.; Zhang, J.; Li, X. J.; Shen, M. H.; Sep. Sci. Technol. 2009, 44, 1884.

50. Strunz, G. M.; Finlay, H.; Tetrahedron 1994, 50, 11113.

51. Mitra, A. K.; De, A.; Karchaudhuri, N.; Synth. Commun. 1999, $29,573$.

52. Concellón, J. M.; Pérez-Andrés, J. A.; Rodríguez-Solla, H.; Chem.--Eur. J. 2001, 7, 3062.

53. Huang, Z. Z.; Wen, L. W.; Huang, X.; Synth. Commun. 1990, 20,2579

54. Schauer, D. J.; Helquist, P.; Synthesis 2006, 3654.

55. Zhang, X. H.; Li, R. L.; Cai, M. S.; Beijing Med. College Trans. 1980, 12, 83. (CA 93:142676).

56. Stepanova, O. S.; Mazurenko, G. A.; Tho, N. V.; Derkach, N.; Fiziologicheski Aktivnye Veshchestva 1976, 8, 86. (CA 86:189422).

57. Xiao, W. J.; Shi, L. L.; Chen, Z. Q.; Huang, Y. Z.; Lang, S. A.; Heteroat. Chem. 1990, 1, 245.

58. Vig, B.; Kanwar, R.; Singh, V.; Indian J. Chem., Sect. B: Org. Chem. Incl. Med. Chem. 1977, 15B, 1048.

59. Delaney, A. D.; Currie, D. J.; Holmes, H. L.; Can. J. Chem. 1969, 47, 3273.

60. Khan, A. M.; Proctor, G. R.; Rees, L.; J. Chem. Soc., C 1966, 990.

61. Homans, A. L.; Fuchs, A.; J. Chromatogr., A 1970, 51, 327.

62. Harmatha, J.; Nawrot, J.; Entomol. Exp. Appl. 2002, 104, 51.

63. Miyazawa, M.; Yoshio, K.; Ishikawa, Y.; Kameoka, H.; J. Agric. Food Chem. 1998, 46, 1914.

Submitted: February 26, 2009

Published online: June 11, 2010

FAPESP helped in meeting the publication costs of this article. 


\section{Antifungal Activity of Natural and Synthetic Amides from Piper species}

\section{Joaquim V. Marques, ${ }^{a}$ Alberto de Oliveira, ${ }^{a, b}$ Ludmila Raggi, ${ }^{c}$ Maria C. M. Young ${ }^{c}$ and Massuo J. Kato ${ }^{a *}$}

${ }^{a}$ Instituto de Química, Universidade de São Paulo, CP 26077, 05513-970 São Paulo- SP, Brazil

${ }^{b}$ Instituto de Química, Universidade Federal de Uberlândia, CP 593, 38400-902 Uberlândia-MG, Brazil

${ }^{c}$ Seção de Fisiologia e Bioquímica de Plantas, Instituto de Botânica, CP 4005, 10051 São Paulo-SP, Brazil

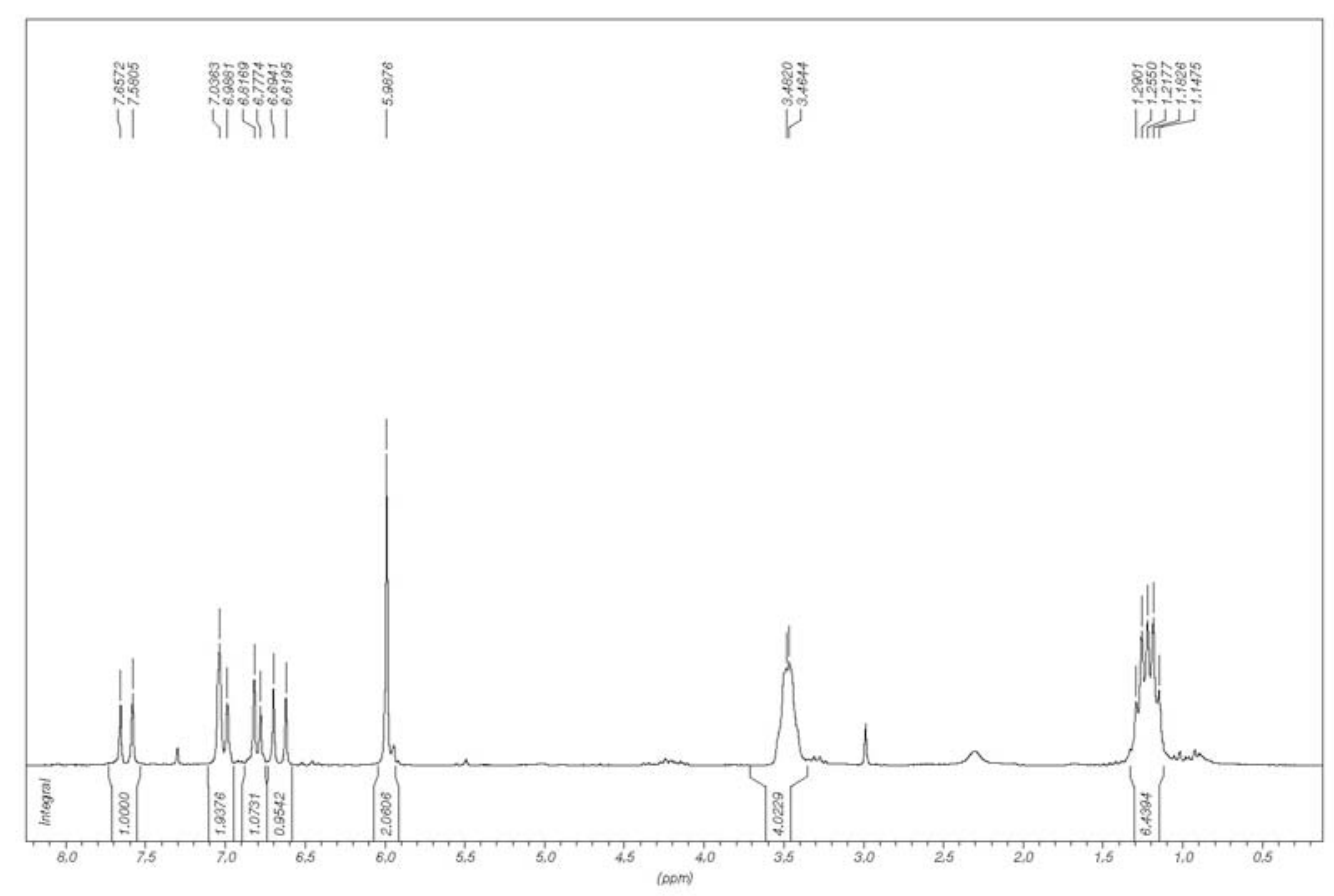

Figure S1. ${ }^{1} \mathrm{H}$ NMR $\left(\mathrm{CDCl}_{3}, 200 \mathrm{MHz}\right)$ of $\mathbf{1 b}$. 


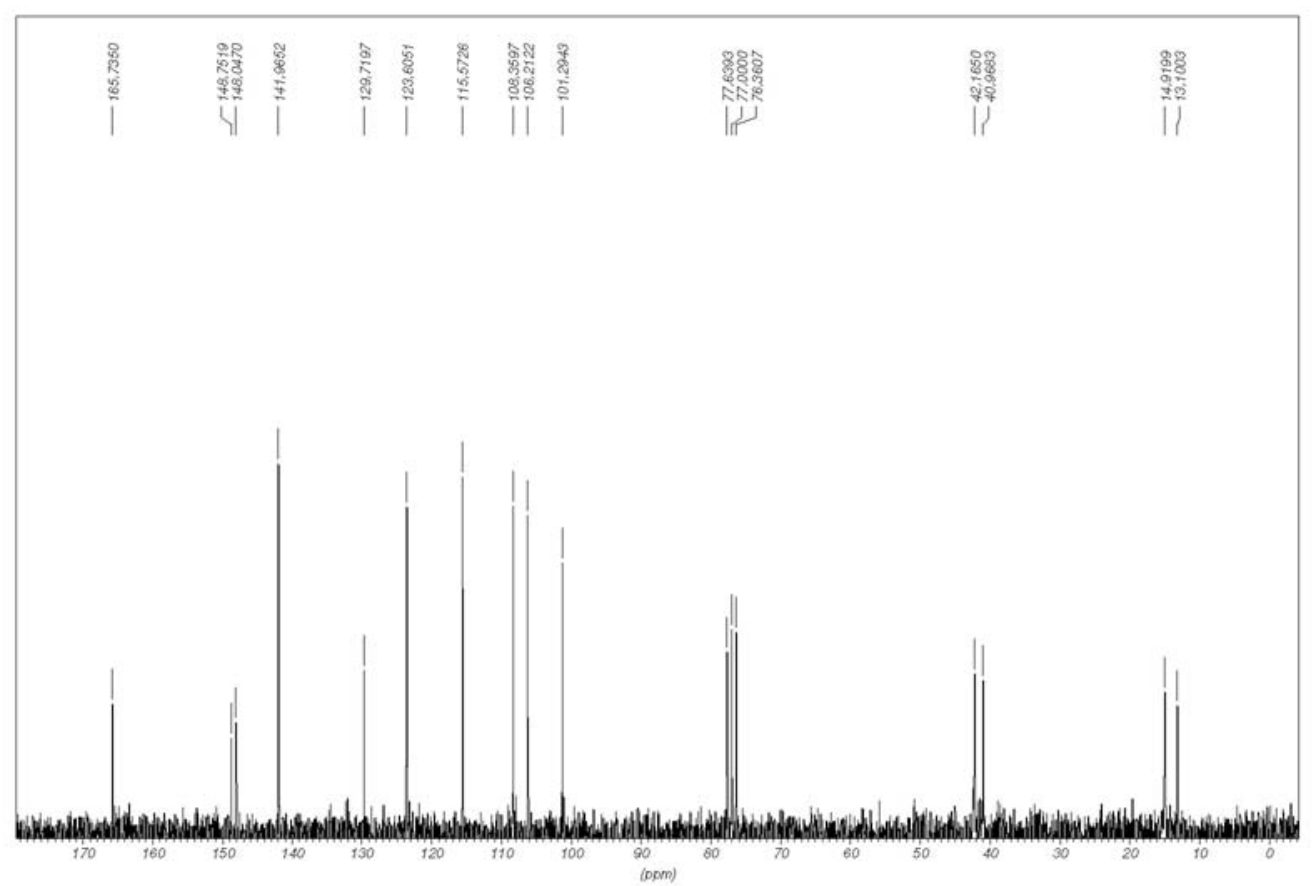

Figure S2. ${ }^{13} \mathrm{C} \mathrm{NMR}\left(\mathrm{CDCl}_{3}, 50 \mathrm{MHz}\right)$ of $\mathbf{1 b}$.

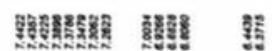

ㄴ.

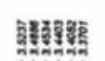

isifint

\section{|}

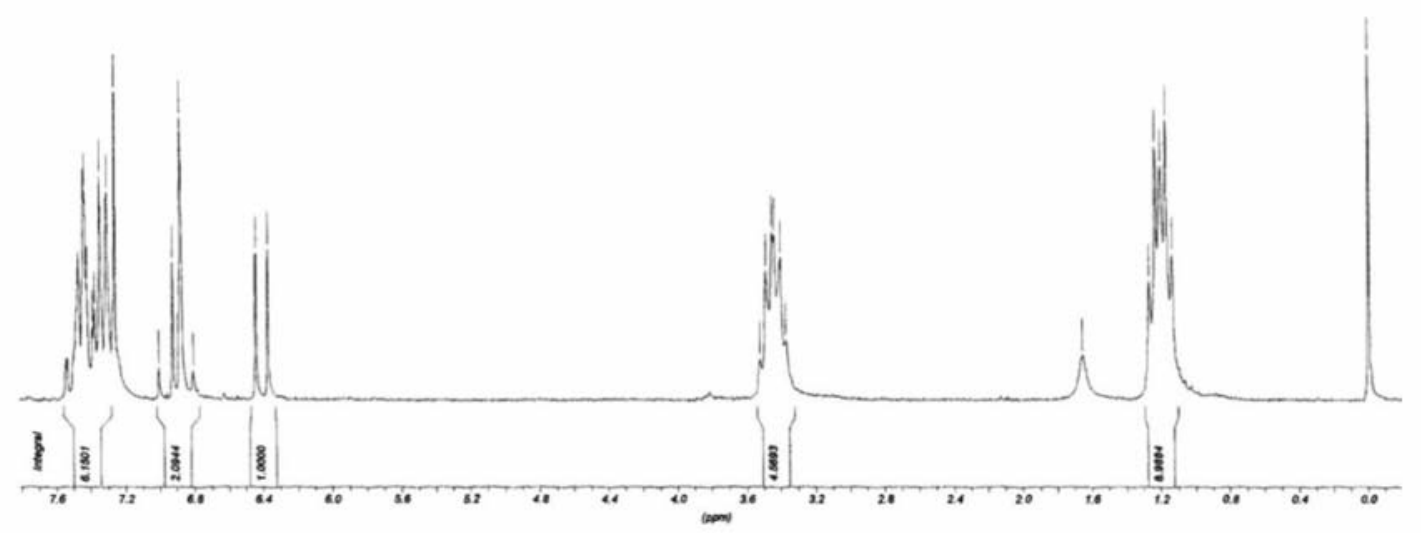

Figure S3. ${ }^{1} \mathrm{H} \mathrm{NMR}\left(\mathrm{CDCl}_{3}, 200 \mathrm{MHz}\right)$ of $\mathbf{1 c}$. 
Vol. 21, No. 10, 2010

Marques et al.

SB

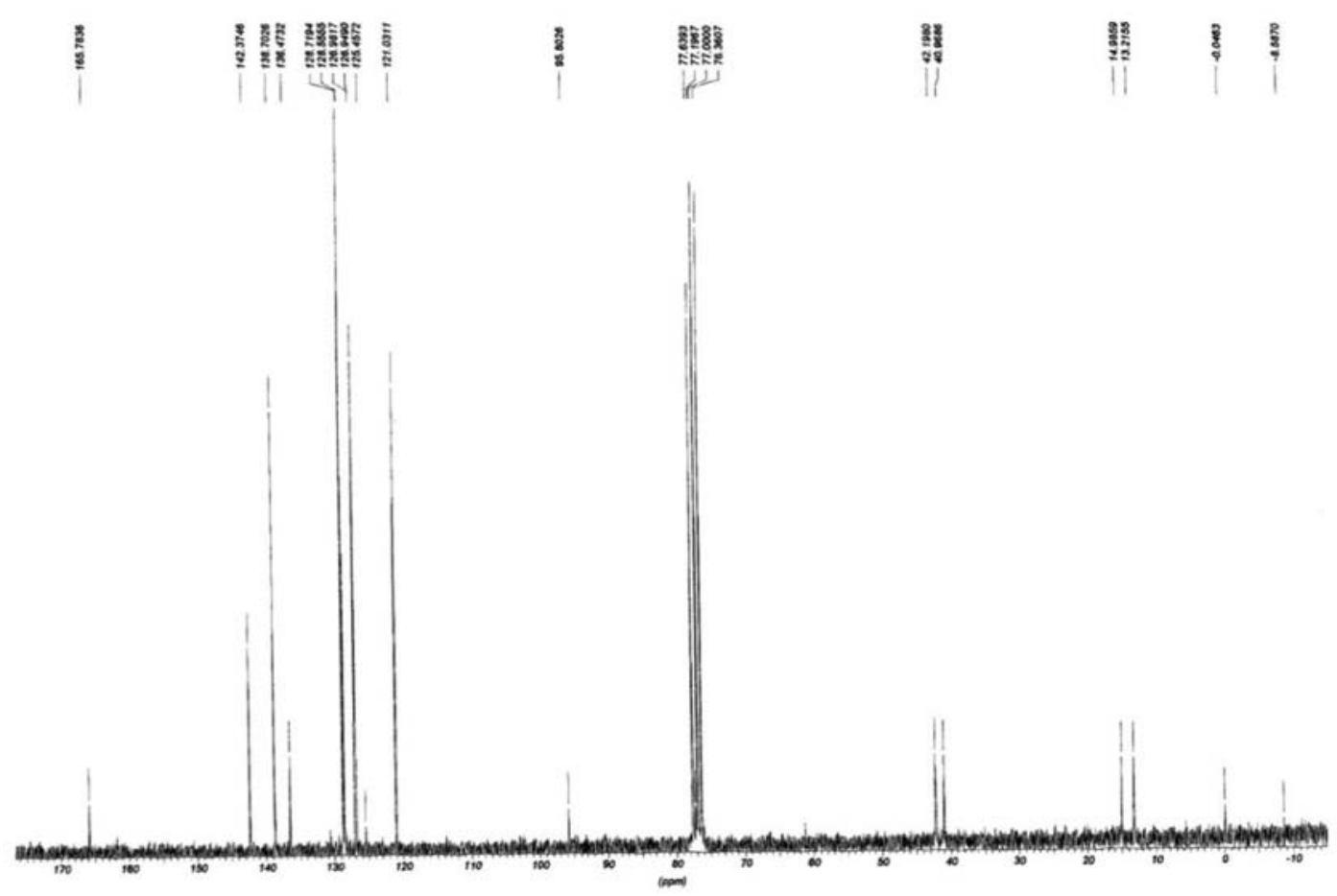

Figure S4. ${ }^{13} \mathrm{C} \mathrm{NMR}\left(\mathrm{CDCl}_{3}, 50 \mathrm{MHz}\right)$ of $\mathbf{1 c}$.

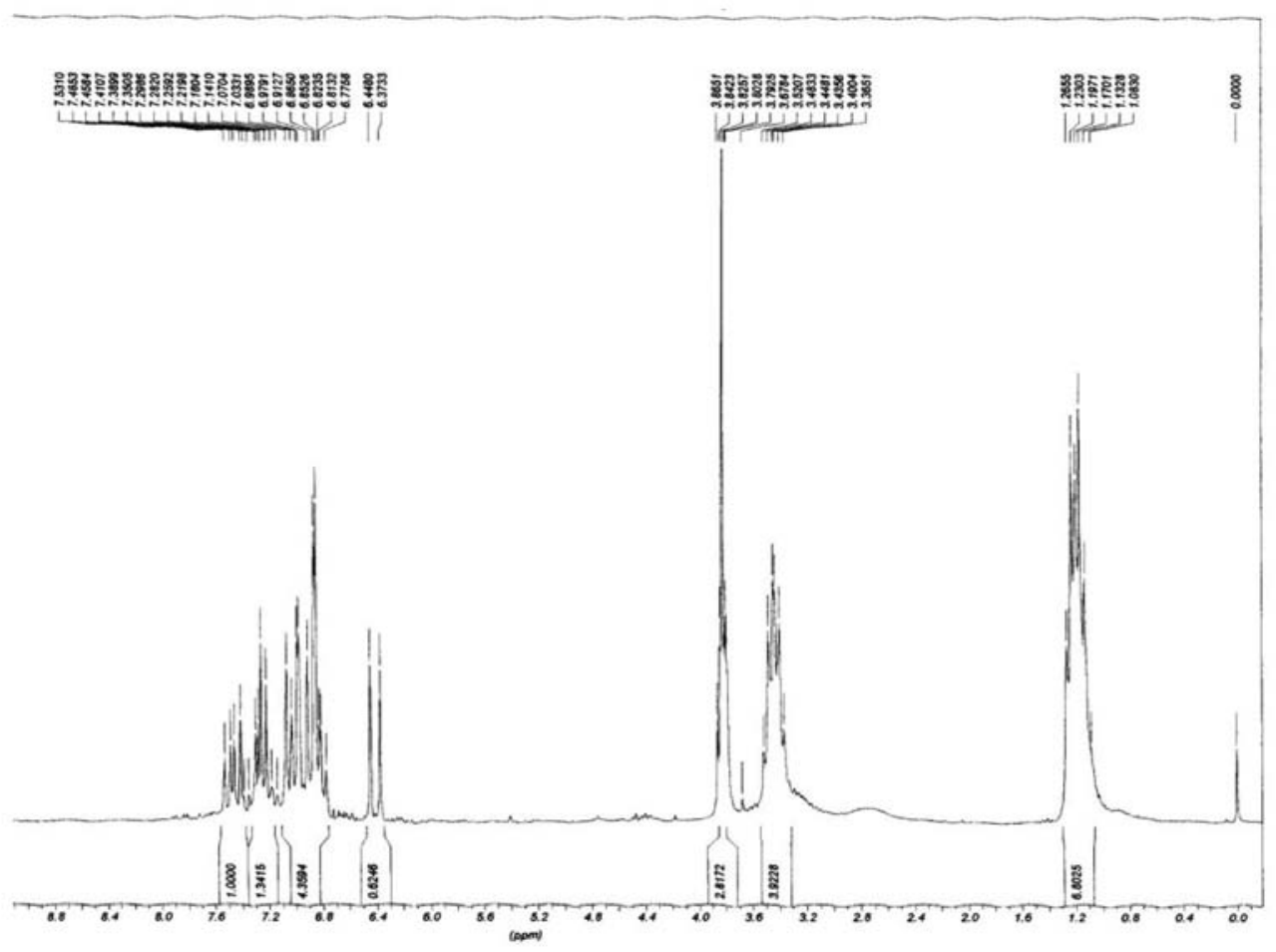

Figure S5. ${ }^{1} \mathrm{H}$ NMR $\left(\mathrm{CDCl}_{3}, 200 \mathrm{MHz}\right)$ of $\mathbf{1 d}$. 


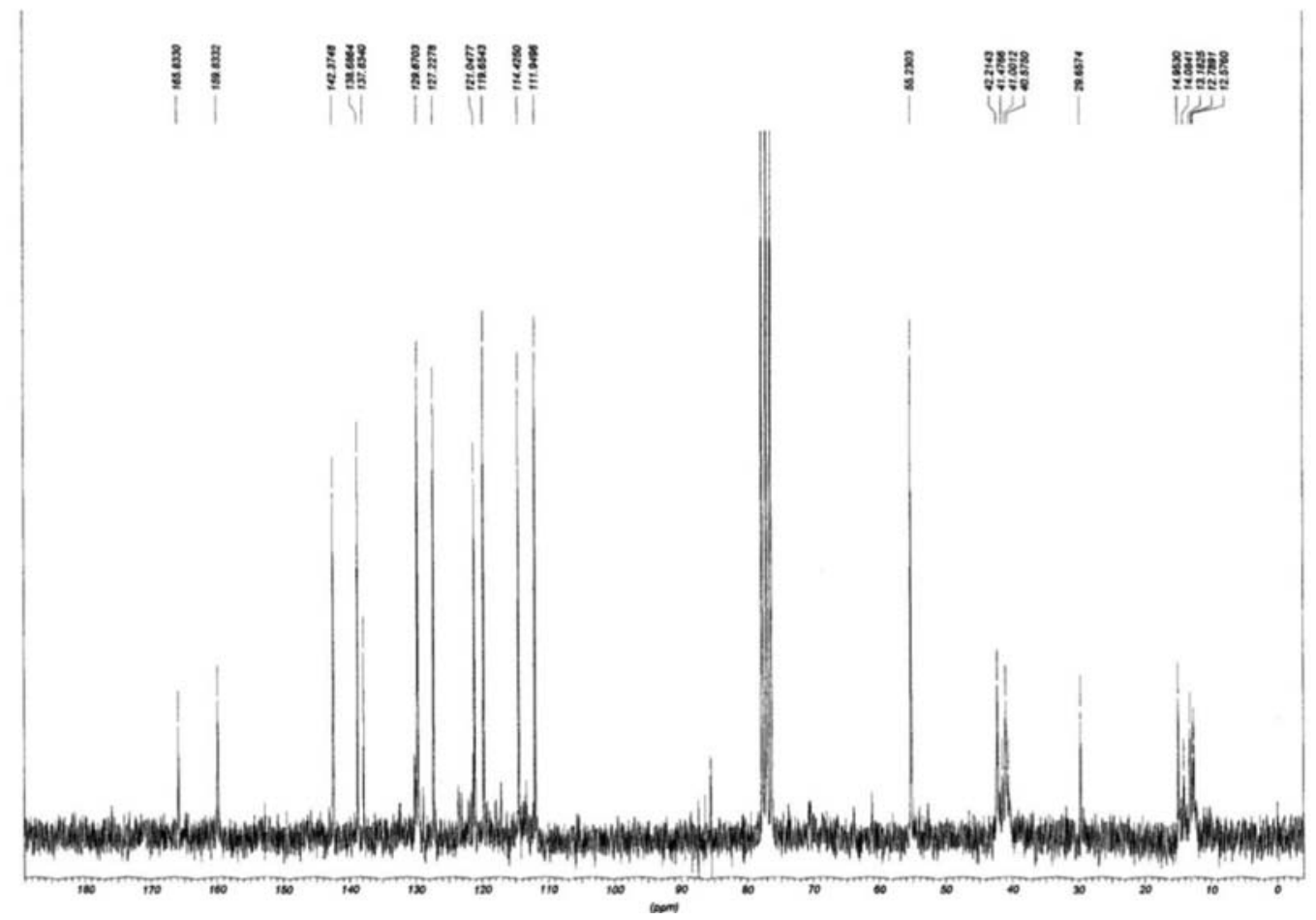

Figure S6. ${ }^{13} \mathrm{C} \mathrm{NMR}\left(\mathrm{CDCl}_{3}, 50 \mathrm{MHz}\right)$ of $\mathbf{1 d}$.

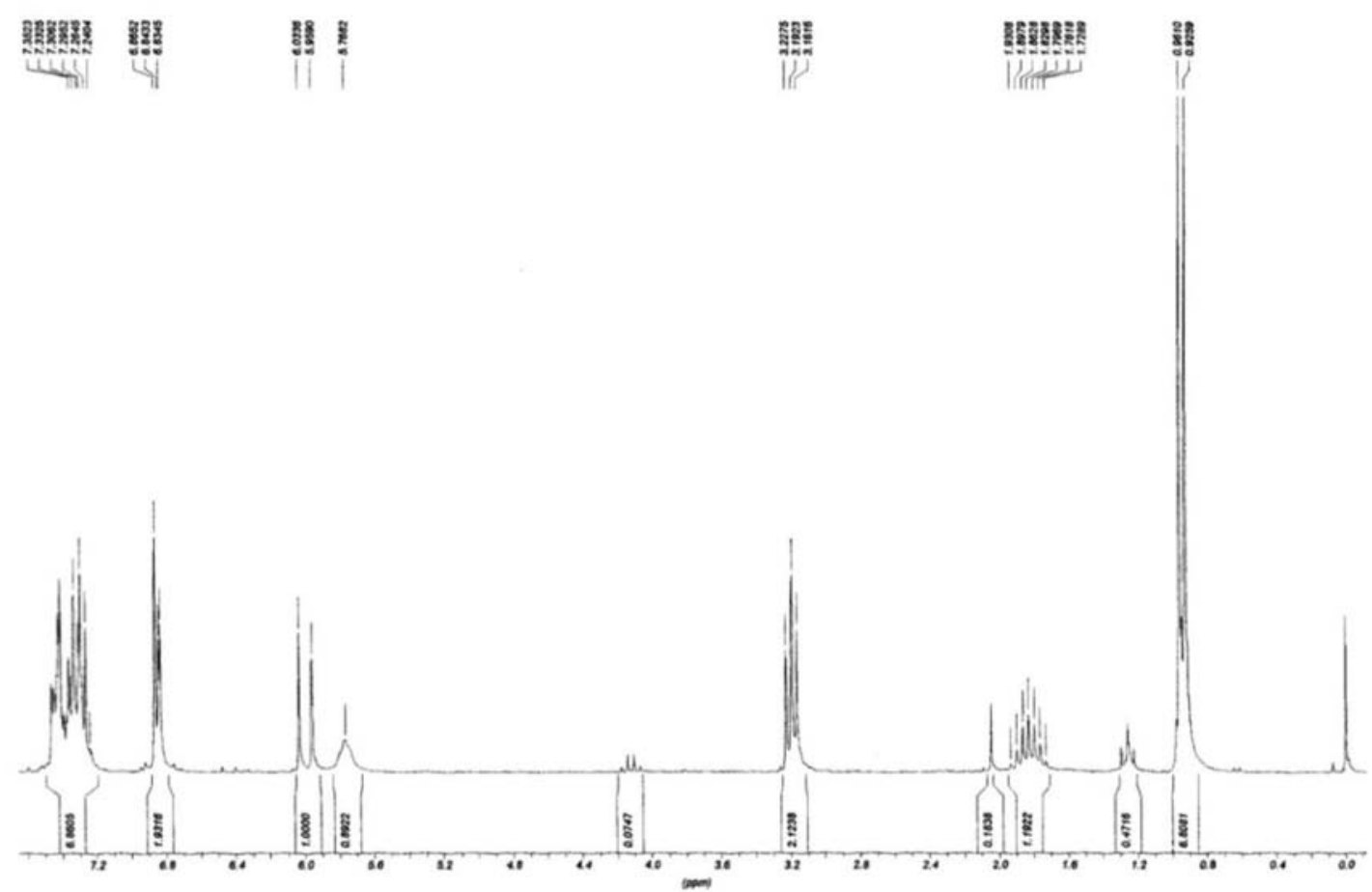

Figure S7. ${ }^{1} \mathrm{H} \mathrm{NMR}\left(\mathrm{CDCl}_{3}, 200 \mathrm{MHz}\right)$ of $\mathbf{2 c}$. 


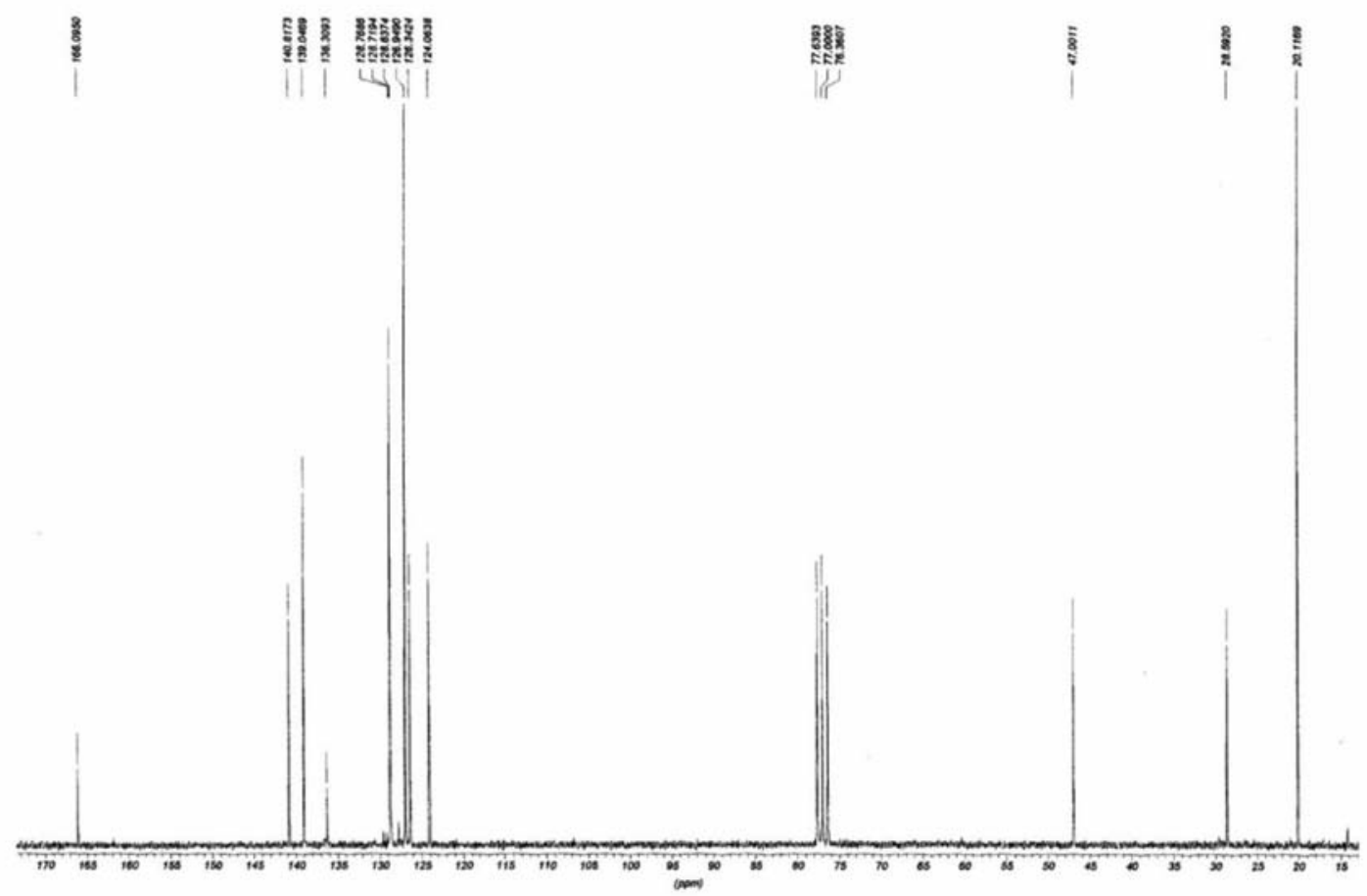

Figure S8. ${ }^{13} \mathrm{C} \mathrm{NMR}\left(\mathrm{CDCl}_{3}, 50 \mathrm{MHz}\right)$ of $\mathbf{2 c}$.

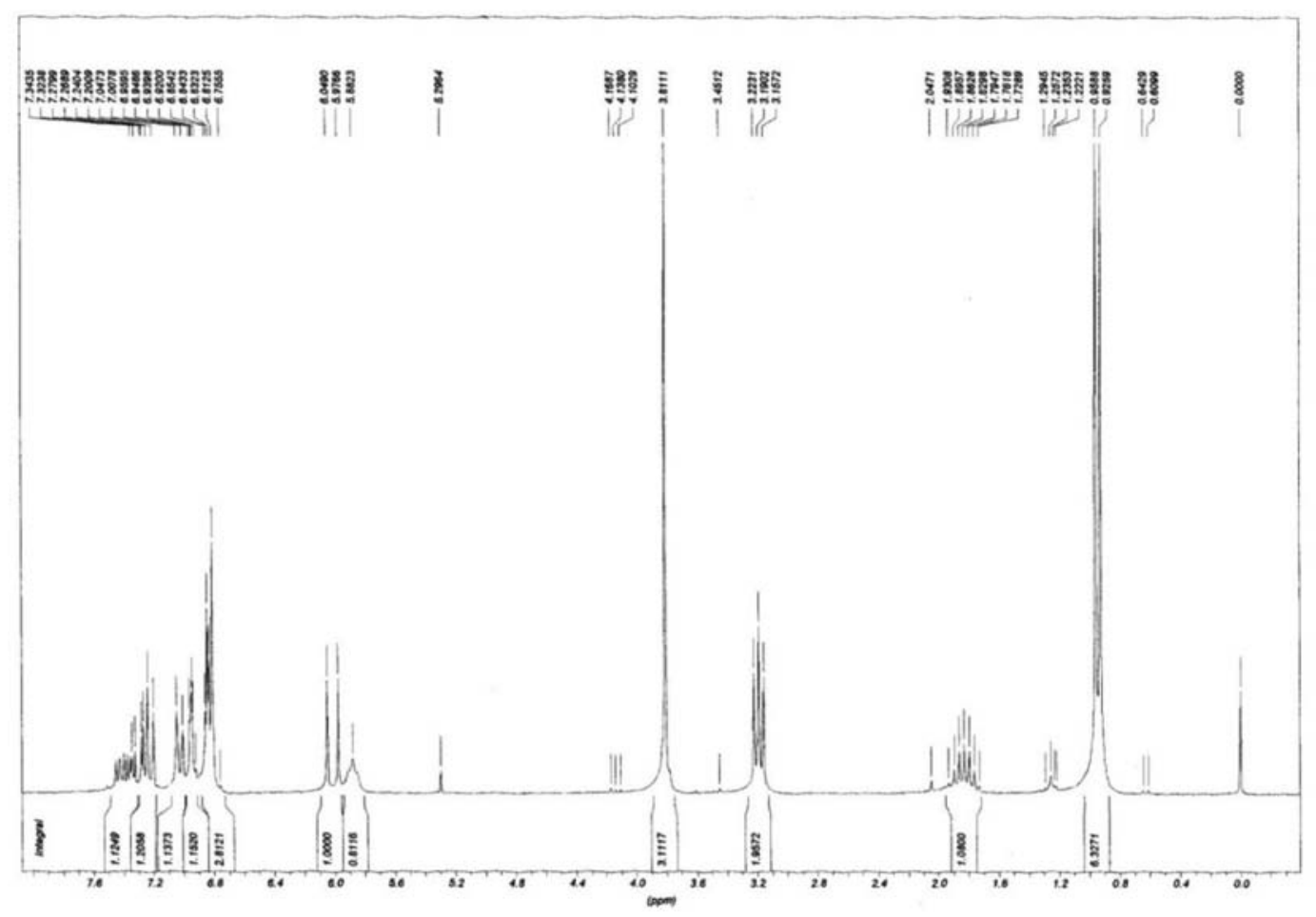

Figure S9. ${ }^{1} \mathrm{H} \mathrm{NMR}\left(\mathrm{CDCl}_{3}, 200 \mathrm{MHz}\right)$ of $\mathbf{2 d}$. 


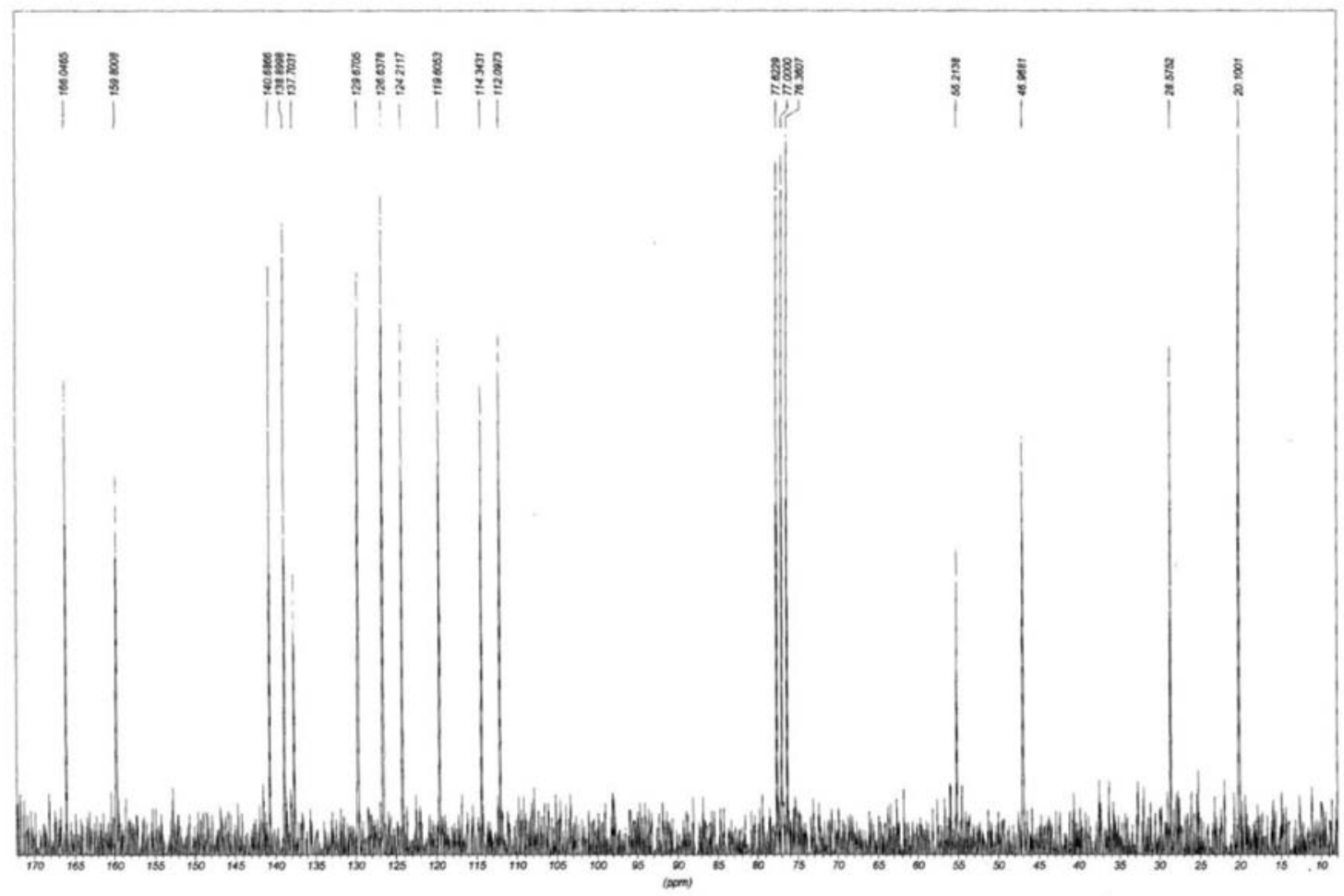

Figure S10. ${ }^{13} \mathrm{C}$ NMR $\left(\mathrm{CDCl}_{3}, 50 \mathrm{MHz}\right)$ of $\mathbf{2 d}$.

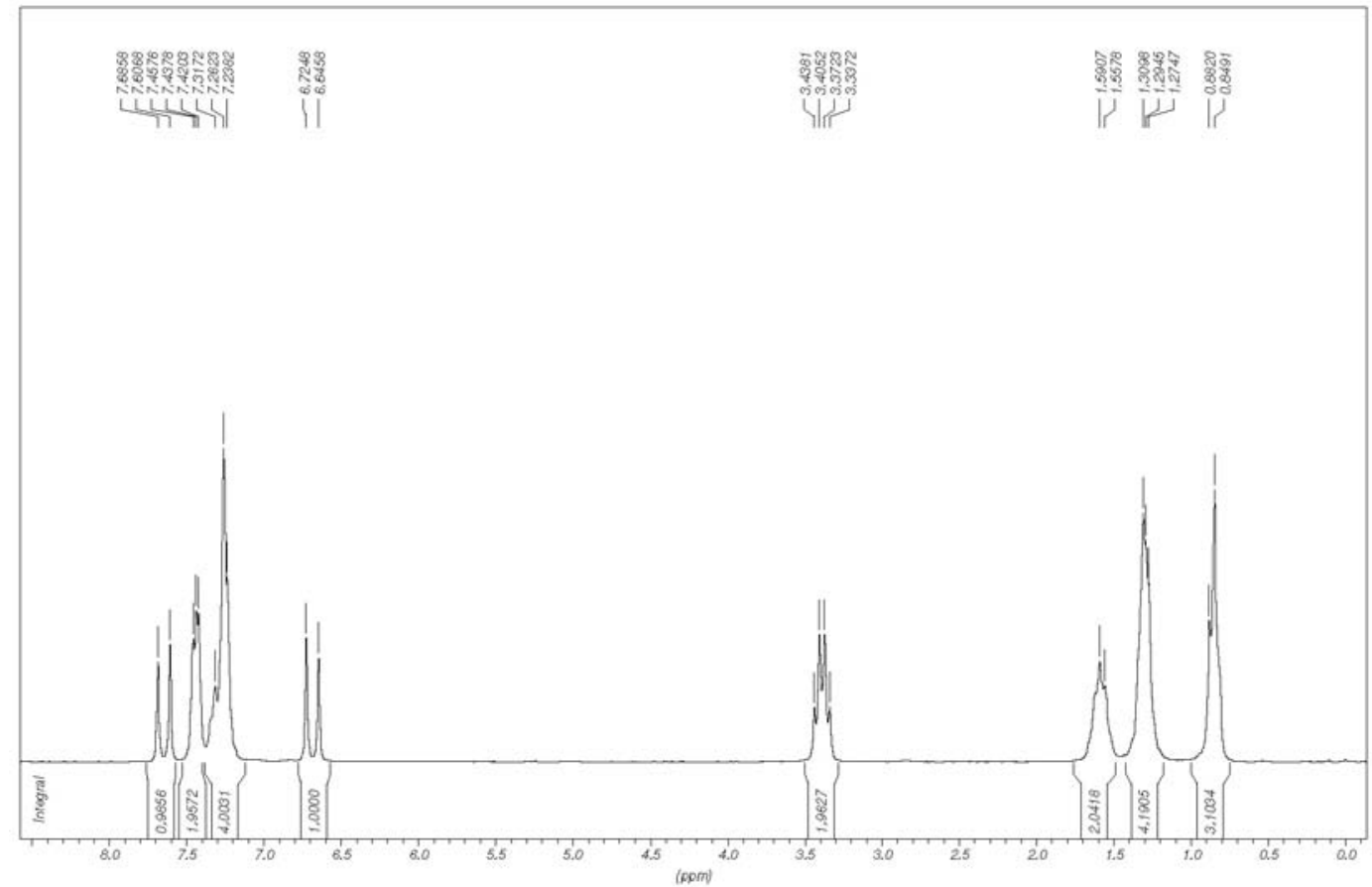

Figure S11. ${ }^{1} \mathrm{H}$ NMR $\left(\mathrm{CDCl}_{3}, 200 \mathrm{MHz}\right)$ of $\mathbf{3 a}$. 


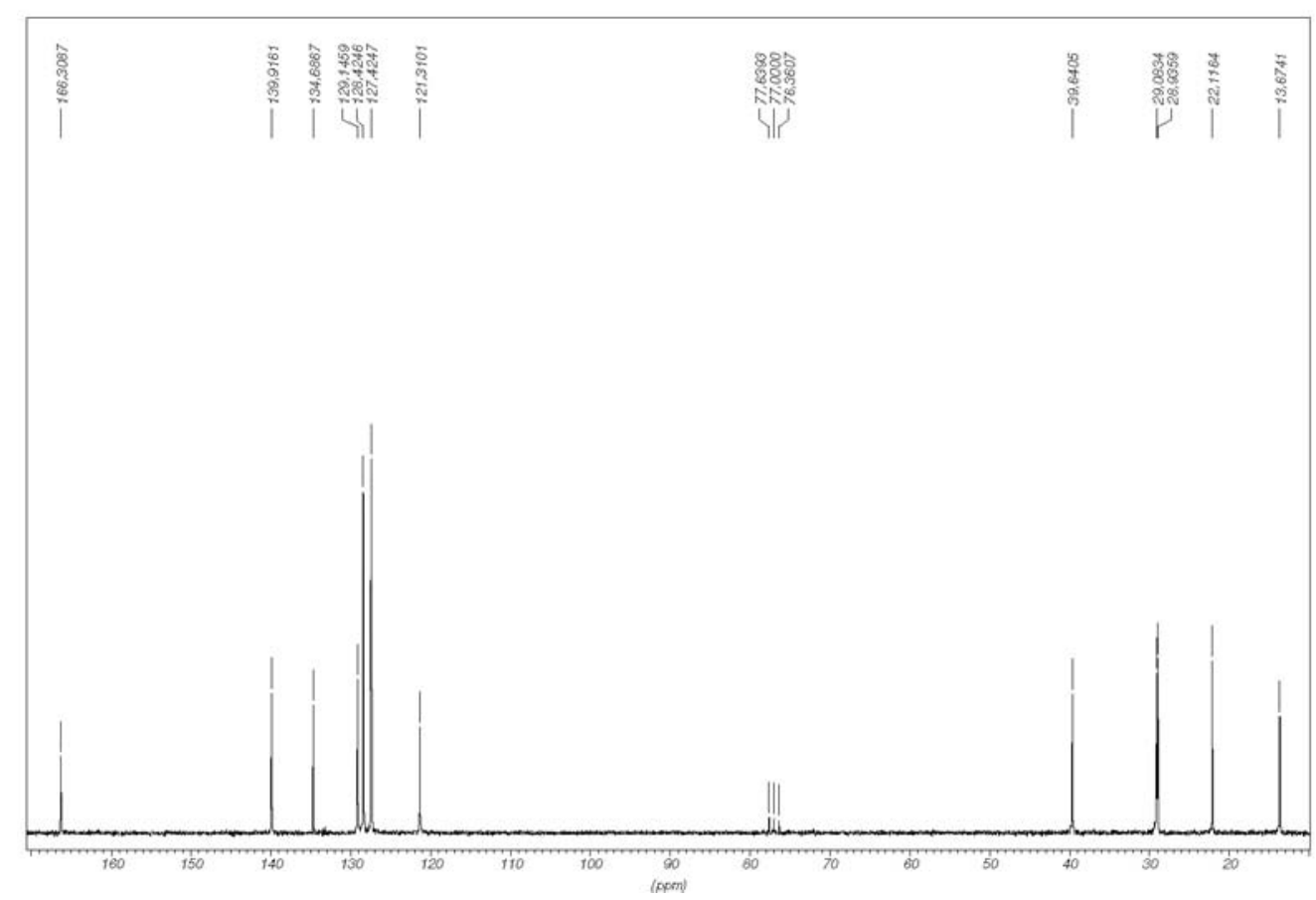

Figure S12. ${ }^{13} \mathrm{C} \mathrm{NMR}\left(\mathrm{CDCl}_{3}, 50 \mathrm{MHz}\right)$ of $\mathbf{3 a}$.

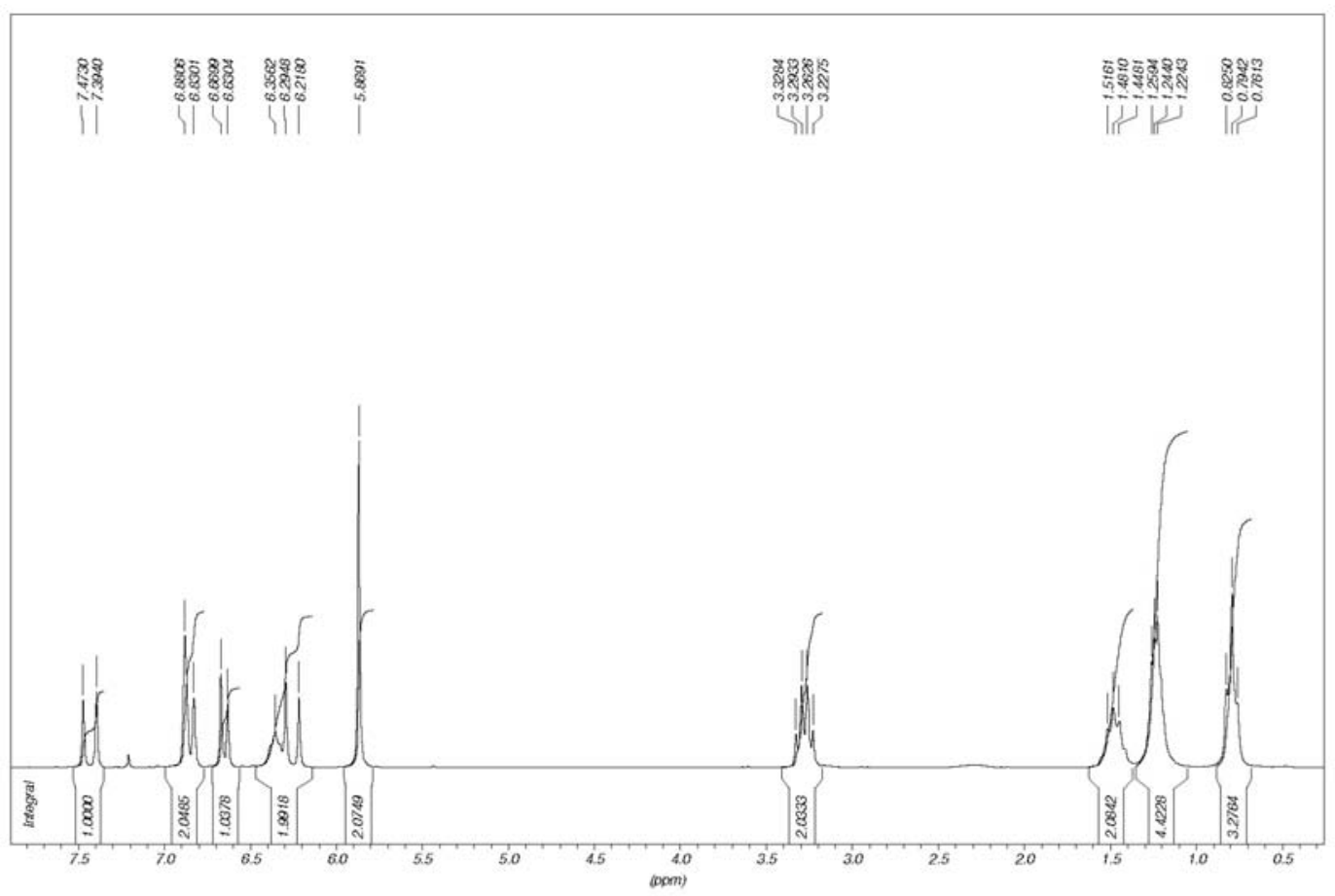

Figure S13. ${ }^{1} \mathrm{H}$ NMR $\left(\mathrm{CDCl}_{3}, 200 \mathrm{MHz}\right)$ of $\mathbf{3 b}$. 


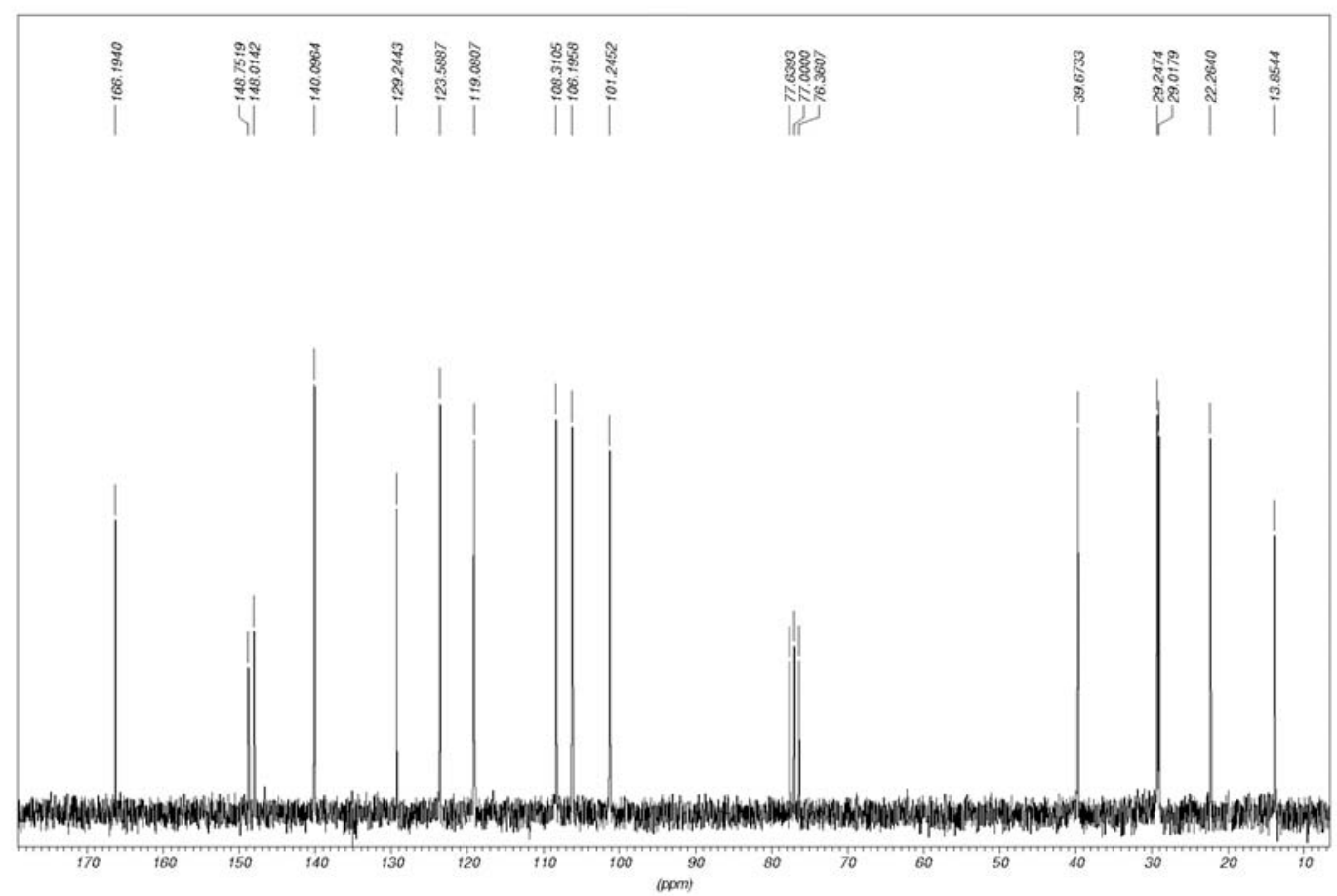

Figure S14. ${ }^{13} \mathrm{C}$ NMR $\left(\mathrm{CDCl}_{3}, 50 \mathrm{MHz}\right)$ of $\mathbf{3 b}$.

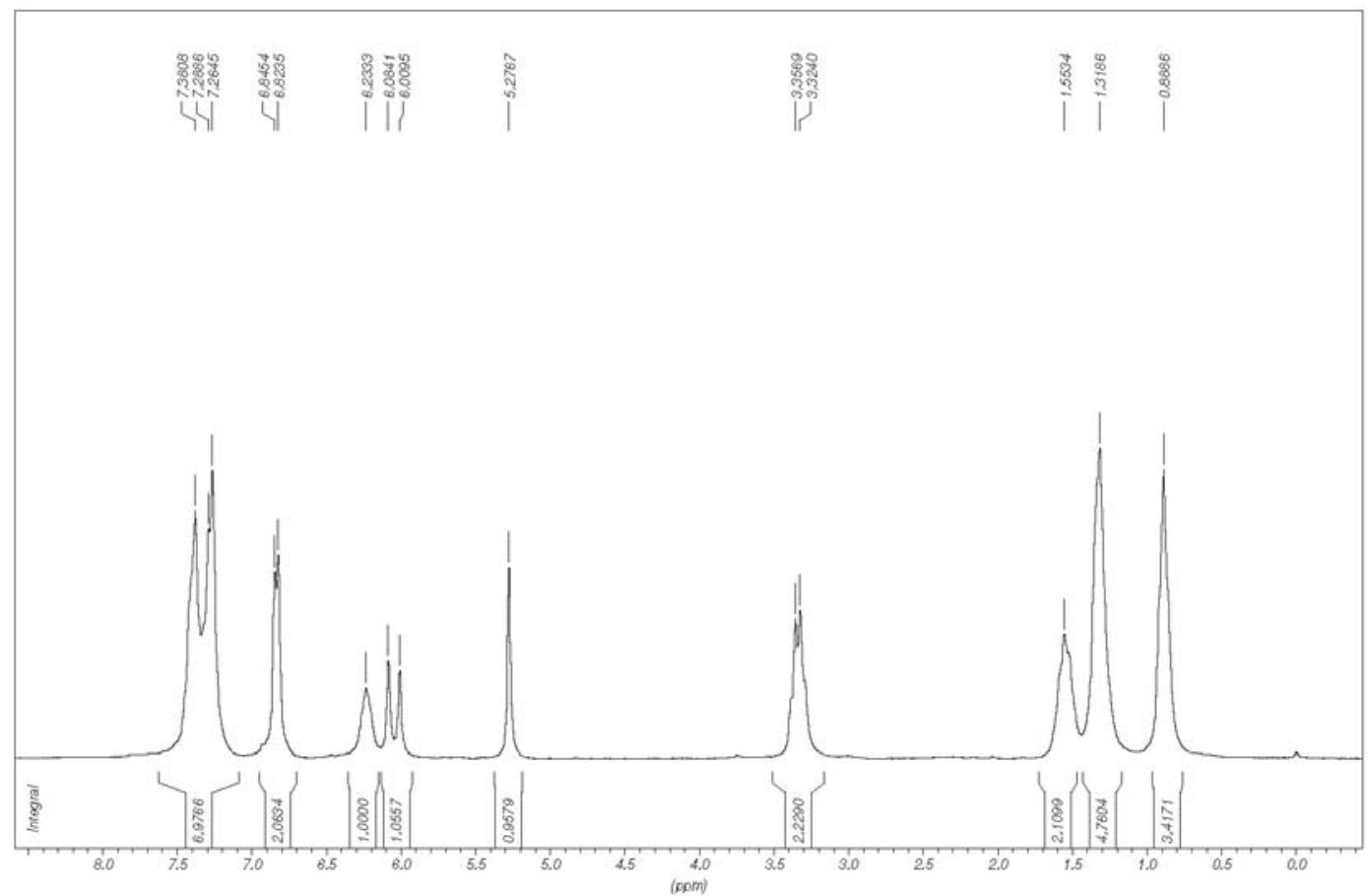

Figure S15. ${ }^{1} \mathrm{H}$ NMR $\left(\mathrm{CDCl}_{3}, 200 \mathrm{MHz}\right)$ of $\mathbf{3 c}$. 


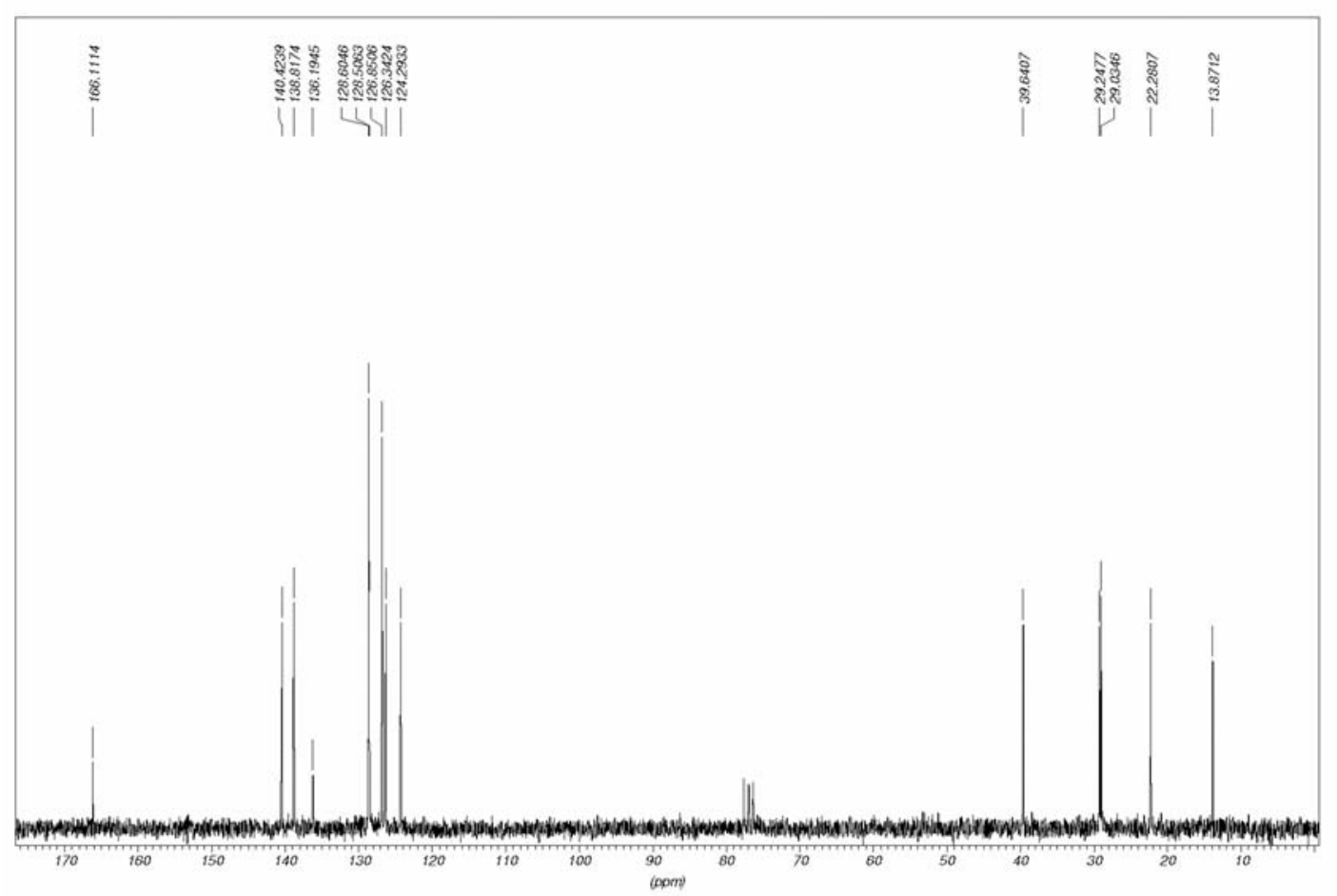

Figure S16. ${ }^{13} \mathrm{C} \mathrm{NMR}\left(\mathrm{CDCl}_{3}, 50 \mathrm{MHz}\right)$ of $\mathbf{3 c}$.

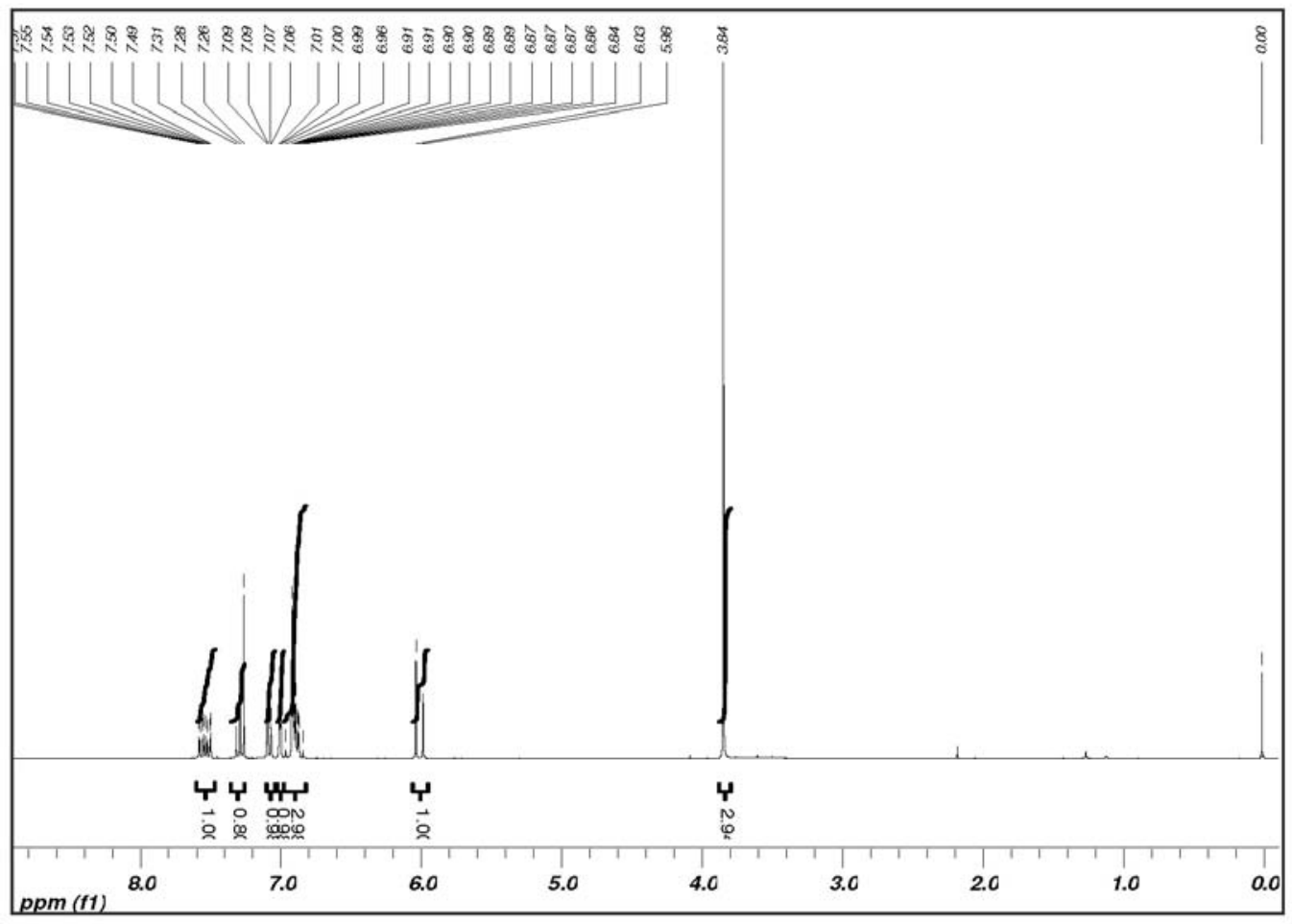

Figure S17. ${ }^{1} \mathrm{H} \mathrm{NMR}\left(\mathrm{CDCl}_{3}, 300 \mathrm{MHz}\right)$ of 7. 


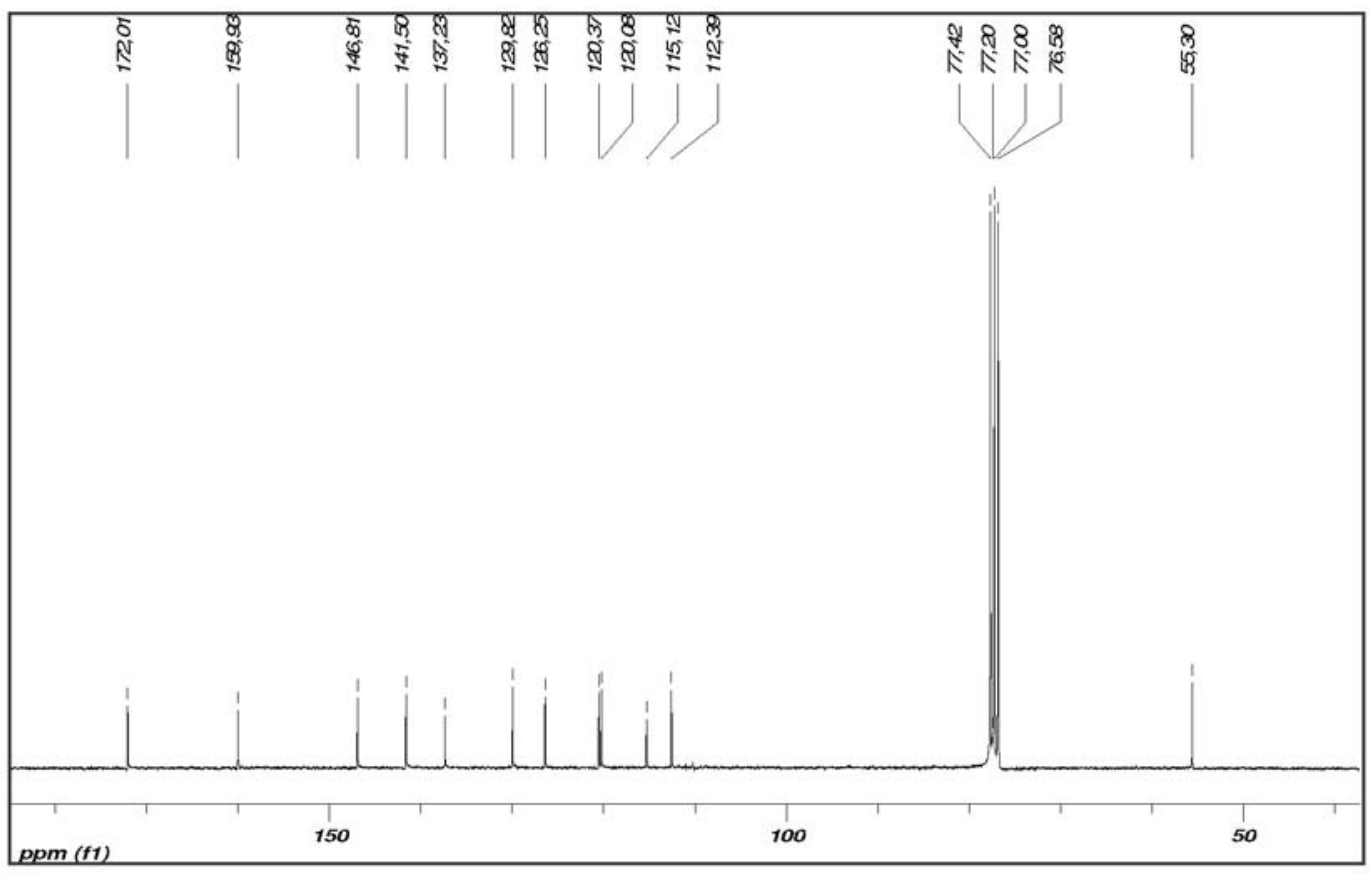

Figure S18. ${ }^{13} \mathrm{C} \mathrm{NMR}\left(\mathrm{CDCl}_{3}, 75 \mathrm{MHz}\right)$ of $\mathbf{7}$. 